\title{
The Growth of CM Periods over False Tate Extensions
}

\section{Daniel Delbourgo and Thomas Ward}

\section{CONTENTS}

\section{Introduction}

2. Theta Lifts and the Katz Measure

3. Calculating Motivic versus Automorphic Periods

4. The Connection with $\Lambda$-Modules

5. Asymptotic Growth in the CM Periods

6. Appendix: Evaluating the $\nabla_{K_{n}^{+}}^{\mathrm{Sym}^{2} E}$ Factors

Acknowledgments

References

2000 AMS Subject Classification: Primary: 11R23; Secondary: 11G40, 19B28

Keywords: Iwasawa theory, complex multiplication, elliptic cures, K-theory, $L$-functions
We prove weak forms of Kato's $\mathrm{K}_{1}$-congruences for elliptic curves with complex multiplication, subject to two technical hypotheses. We next use MAGMA to calculate the $\mu$-invariant measuring the discrepancy between the "motivic" and "automorphic" $p$-adic $L$-functions. Via the two-variable main conjecture, one can then estimate growth in this $\mu$-invariant using arithmetic of the $\mathbb{Z}_{p}^{2}$-extension.

\section{INTRODUCTION}

Over the last decade or so, the generalization of K. Iwasawa's ideas to nonabelian extensions of number fields has yielded a host of conjectures in mathematics. Thanks to the recent efforts of Kato, Ritter-Weiss, Hara, and Kakde, many of the "main conjectures" in this burgeoning subject have been converted into theorems. A key feature common to all the methods of these researchers is the fact that Artin $L$-values occur as constant terms in the $q$-expansion of $\Lambda$-adic Eisenstein series (therefore, to find congruences among $L$-values, it is enough to find congruences within the $q$-expansions).

It is natural to ask what happens if we replace Tate motives by elliptic curves. In [Delbourgo and Ward 08], we established certain $\mathrm{K}_{1}$-congruences between critical values of the Hasse-Weil $L$-function over the finite layers in a false Tate extension. We were forced to assume that the elliptic curve in question was semistable, due to technical difficulties on the automorphic side. At that time, we were on the lookout for new elliptic curves that might yield to complementary methods; an appropriate place to search for specimens is the theory of complex multiplication.

Currently, there are two approaches to proving nonabelian congruences: the Rankin-Selberg method and $\Lambda$-adic Eisenstein series on unitary groups.

Thanasis Bouganis has made considerable progress employing the second approach; he aims to realize the Hasse-Weil $L$-value as the constant term in an Eisenstein series on $\mathrm{GU}(n, n)$ and hopes that the appropriate $q$-expansions will yield congruences.

(C) A K Peters, Ltd. 1058-6458/2010 \$0.50 per page Experimental Mathematics 19:2, page 195 
In this article we will study the first approach, namely the Rankin-Selberg method. While it appears (superficially) more tractable, one soon encounters an annoying error term measuring the failure of the Petersson inner product to coincide with the Néron period. This period ratio grows as we climb the tower of totally real fields, and one of the major tasks (in this paper) is to compute the growth rate precisely.

Let $E$ be an elliptic curve defined over $\mathbb{Q}$ admitting complex multiplication by an order in the $\operatorname{ring} \mathcal{O}_{K}$, where $K=\mathbb{Q}(\sqrt{-D})$ denotes an imaginary quadratic field. Fix a prime number $p \neq 2$ that splits into $(p)=\mathfrak{p} \times \mathfrak{p}^{*}$ inside $\mathcal{O}_{K}$, so that $E$ possesses good ordinary reduction over $\mathbb{Q}_{p}$. We also pick an auxiliary $p$-power-free integer $\Delta>1$ that is coprime to $p$ and to the conductor $N_{E}$ of the elliptic curve.

If one considers the false Tate extension $K_{\mathrm{FT}}=$ $\bigcup_{n \geq 1} \mathbb{Q}\left(\sqrt{-D}, \mu_{p^{n}}, \sqrt[p n]{\Delta}\right)$, elementary Galois theory tells us that

$$
G_{\infty}:=\operatorname{Gal}\left(K_{F T} / K\right) \cong\left(\begin{array}{cc}
\mathbb{Z}_{p}^{\times} & \mathbb{Z}_{p} \\
0 & 1
\end{array}\right)
$$

is a noncommutative $p$-adic Lie group of dimension 2 . Writing $K_{n}=\mathbb{Q}\left(\sqrt{-D}, \mu_{p^{n}}\right)$, then $\rho_{n}=\operatorname{Ind}_{K_{n}}^{K}\left(\chi_{n}\right)$ will denote the unique symplectic representation of $G_{\infty}$ of degree $p^{n}-p^{n-1}$. The irreducible Artin representations of $G_{\infty}$ are all of the form $\rho_{n} \otimes \psi$ for some $n \geq 0$ and some finite-order character $\psi: \operatorname{Gal}\left(K\left(\mu_{p^{\infty}}\right) / K\right) \rightarrow \mathbb{C}^{\times}$.

Finally, the Galois group of $K\left(\mu_{p^{\infty}}\right)$ over $K_{n}$ will be abbreviated by $U^{(n)}$.

Definition 1.1. The motivic p-adic L-function $\mathcal{L}\left(E, \rho_{n}\right)$ of $E_{/ K}$ twisted by $\rho_{n}$ is the unique element of $\mathbb{Z}_{p} \llbracket U^{(n)} \rrbracket \otimes$ $\mathbb{Q}$ satisfying

$$
\begin{aligned}
\psi\left(\mathcal{L}\left(E, \rho_{n}\right)\right)= & \frac{\epsilon\left(\rho_{n} \otimes \psi\right)_{p}}{\alpha_{p}^{\mathfrak{f} \psi}} \times\{\text { Euler factor at } p\} \\
& \times \frac{L_{\{p \Delta\}}\left(E / K, \rho_{n} \otimes \psi^{-1}, 1\right)}{\left(\Omega_{E}^{+} \Omega_{E}^{-}\right)^{\left[K_{n}: K\right]}}
\end{aligned}
$$

at all Dirichlet characters $\psi: U^{(n)} \rightarrow \overline{\mathbb{Q}}_{p}^{\times}$of conductor $\mathfrak{f}_{\psi}$.

The existence itself follows from interpolation properties of the Katz-Eisenstein measure, which is discussed in the next section. We simply point out that $\epsilon\left(\rho_{n} \otimes \psi\right)_{p}$ is the $p$-part of the $\epsilon$-factor suitably normalized as in [Coates et al. 05] (the modification of the $p$-Euler factor is also described later on).
To make further progress, we must now make two assumptions.

Hypothesis 1.2. For each integer $j \in\{0, \ldots, n\}$, [Stevens 89, Conjecture IV, Section 4] holds at all $\rho_{j} \otimes \psi$ twists of the $f_{E}$-isotypic component in $H_{1}\left(X_{1}\left(N_{E}\right), \mathbb{Z}\right)$.

Hypothesis 1.3. Each analytic $\mu$-invariant associated to the $(p-1)$-branches of the Mazur-Tate-Teitelbaum $p$-adic $L$-function for $E / K$ vanishes.

Hypothesis 1.2 implies $p$-integrality of the motivic $p$-adic $L$-functions $\mathcal{L}\left(E, \rho_{0}\right), \mathcal{L}\left(E, \rho_{1}\right), \ldots, \mathcal{L}\left(E, \rho_{n}\right)$ from Definition 1.1. For instance, if the $\rho_{j}$-twisted main conjectures hold for $j \in\{0, \ldots, n\}$, then every $\mathcal{L}\left(E, \rho_{j}\right)$ is the characteristic power series of a corresponding Selmer group, in which case Hypothesis 1.2 follows.

The second condition is basically equivalent to $\operatorname{Norm}_{0,1}\left(\mathcal{L}\left(E, \rho_{0}\right)\right)$ exhibiting a trivial $\mu$-invariant (note that for all positive integers $j \leq n$, the norm map induces a homomorphism $\operatorname{Norm}_{j, n}: \mathbb{Z}_{p} \llbracket U^{(j)} \rrbracket \rightarrow \mathbb{Z}_{p} \llbracket U^{(n)} \rrbracket$ on the completed group rings). This triviality condition can always be checked numerically by computing $L$-values; it is true, for example, whenever the leading term of this norm power series is a $p$-adic unit.

Theorem 1.4. Assume that Hypothesis 1.2 holds for a given $n \in \mathbb{N}$ and also that Hypothesis 1.3 is true. Then there is a family of congruences

$$
\begin{aligned}
\mathcal{L}\left(E, \rho_{n}\right) & \equiv \operatorname{Norm}_{n-1, n}\left(\mathcal{L}\left(E, \rho_{n-1}\right)\right) \equiv \cdots \\
& \equiv \operatorname{Norm}_{0, n}\left(\mathcal{L}\left(E, \rho_{0}\right)\right) \quad \bmod p
\end{aligned}
$$

In particular, if $\omega^{i}\left(\mathcal{L}\left(E, \rho_{0}\right)\right) \in \mathbb{Z}_{p}^{\times}$for all $i \in$ $\{0, \ldots, p-2\}$, then by Definition 1.1, clearly one has $L\left(E / K_{1}, 1\right) \neq 0$. Moreover, from Theorem 1.4 it follows directly that

$$
L\left(E / K, \rho_{j}, 1\right) \neq 0 \quad \text { at every } 1 \leq j \leq n .
$$

The observation that the analytic rank of these twists is even can also be deduced from a nice formula in [Coates et al. 10]. However, this nonvanishing result is a bit stronger.

Let $\varphi: \mathbb{Z}_{p} \llbracket U^{(j-1)} \rrbracket \rightarrow \mathbb{Z}_{p} \llbracket U^{(j)} \rrbracket$ be the homomorphism induced by the $p$-power map on $U^{(0)} \cong \mathbb{Z}_{p}^{\times}$. Setting $a_{j}=\mathcal{L}\left(E, \rho_{j}\right)$, one may then define

$$
c_{j}:=\frac{a_{j} \times \varphi \circ \operatorname{Norm}_{0, j-1}\left(a_{0}\right)}{\operatorname{Norm}_{0, j}\left(a_{0}\right) \times \varphi\left(a_{j-1}\right)},
$$


which belongs to the field of quotients $\operatorname{Frac}\left(\mathbb{Z}_{p} \llbracket U^{(j)} \rrbracket\right)$.

Theorem 1.5. Under the same conditions as the previous result,

$$
\prod_{j=1}^{n} \operatorname{Norm}_{j, n}\left(c_{j}\right)^{p^{j}} \equiv 1 \bmod p^{n+1} \cdot \mathbb{Z}_{p}\left[\left[U^{(n)}\right]\right] .
$$

In fact, were these congruences to hold modulo $p^{2 n}$ rather than modulo $p^{n+1}$, then one would establish the existence of a nonabelian $p$-adic $L$-function inside $\mathrm{K}_{1}\left(\mathbb{Z}_{p}\left[\left[G_{\infty}\right]\right]_{\mathcal{S}}\right)$, as explained in [Kato 05, Section 1].

Using different techniques, we obtained analogous congruences in [Delbourgo and Ward 08] for semistable elliptic curves. The advantage of working in the CM case is that verification of Hypotheses 1.2 and 1.3 reduces to calculating Hecke $L$-series over $\mathbb{Q}$, while over $K_{n}^{+}$, conductors of the $l$-adic representations become gigantic.

Remark 1.6. It should be noted that Theorem 1.5 is a rather straightforward consequence of Theorem 1.4, courtesy of a strong mathematical induction argument. The full details are identical to [Delbourgo and Ward 08, Section 3.3], and we shall not reproduce them here. This leaves us with the task of proving Theorem 1.4 in the next section.

Let us now consider Hida's automorphic $p$-adic $L$ function (see Theorem 2.3). This object $\mathcal{L}^{\text {Hida }}\left(E, \rho_{n}\right)$ interpolates exactly the same data as $\mathcal{L}\left(E, \rho_{n}\right)$, except that the period in its denominator is the Petersson selfproduct for the base-change of $f_{E}$ to $K_{n}^{+}$, and there is an extra fudge-factor arising from the congruence module.

If

$$
\Omega_{K_{n}^{+}}^{\operatorname{mot}}(E)=\left(\Omega_{E}^{+} \Omega_{E}^{-}\right)^{\left[K_{n}^{+}: \mathbb{Q}\right]}
$$

and

$$
\Omega_{K_{n}^{+}}^{\mathrm{aut}}(E)=\pi^{\left[K_{n}: \mathbb{Q}\right]}\left\langle\mathbf{B C}_{K_{n}^{+}}\left(f_{E}\right), \mathbf{B C}_{K_{n}^{+}}\left(f_{E}\right)\right\rangle,
$$

then one has the relationship

$$
\mathcal{L}\left(E, \rho_{n}\right)=\frac{\Omega_{K_{n}^{+}}^{\text {aut }}(E)}{\Omega_{K_{n}^{+}}^{\operatorname{mot}}(E)} \times \mathbb{H}_{\lambda}(\underline{2})^{-1} \times \mathcal{L}^{\text {Hida }}\left(E, \rho_{n}\right),
$$

(up to a $p$-adic unit), where $\mathbb{H}_{\lambda}(\underline{2})$ denotes the value of the characteristic power series for the associated congruence module " $C_{0}\left(\lambda_{E, K_{n}^{+}}\right)$," evaluated at parallel weight two (see Section 2 for details). The $p$-adic $L$-function $\mathcal{L}^{\text {Hida }}\left(E, \rho_{n}\right)$ arises from the Rankin convolution approach, and is heavily $p$-integral. However, it is indisputably the natural object to work with from an automorphic point of view.
Remark 1.7. In order to prove the full $\mathrm{K}_{1}$-congruences of Kato, we first need to understand the analytic invariants

$$
p^{\mu_{p, n}^{\mathrm{Per}}(E)}:=\left|\frac{\Omega_{K_{n}^{+}}^{\operatorname{mot}}(E)}{\Omega_{K_{n}^{+}}^{\text {aut }}(E)}\right|_{p}^{-1}
$$

and

$$
p^{\mu_{p, n}^{\mathrm{anti}}(E)}:=\left|\mathbb{H}_{\lambda}(2, \ldots, 2)\right|_{p}^{-1}
$$

and their rate of growth as the fields $K_{n}^{+}$climb up the cyclotomic $\mathbb{Z}_{p^{-}}$extension. Henceforth we shall assume that $p>3$ does not divide the degree of $X_{0}\left(N_{E}\right) \stackrel{\varphi_{E}}{\rightarrow} E$.

One should now consider the two-variable Iwasawa module $\operatorname{Gal}\left(\mathcal{M}_{\infty} / K\left(E\left[p^{\infty}\right]\right)\right)$ with $\mathcal{M}_{\infty}$ the maximal abelian pro- $p$-extension of $K\left(E\left[p^{\infty}\right]\right)$ unramified outside $\mathfrak{p}$. Let us define nonnegative integers

$$
\mu_{E}^{\mathrm{cy}}:=\sum_{j=0}^{p-2} \mu_{\omega^{j}}\left(\mathcal{Z}_{\infty,+}\right) \quad \text { and } \quad \lambda_{E}^{\mathrm{cy}}:=\sum_{j=0}^{p-2} \lambda_{\omega^{j}}\left(\mathcal{Z}_{\infty,+}\right),
$$

where $\left(\mu_{\omega^{j}}, \lambda_{\omega^{j}}\right)$ refer to the " $\mu$-" and " $\lambda$-invariants" for the $\omega_{K}^{j}$-eigenspace inside

$$
\begin{aligned}
& \mathcal{Z}_{\infty,+} \\
& \quad:=H^{0}\left(K(E[p]) / K_{1},\left(\operatorname{Gal}\left(\mathcal{M}_{\infty} / K\left(E\left[p^{\infty}\right]\right)\right) \otimes \Phi_{E, \mathfrak{p}}^{\otimes-2}\right)_{\Gamma_{-}}\right) .
\end{aligned}
$$

Theorem 1.8. Assume that the $\mu_{\omega^{j}}\left(\mathcal{Z}_{\infty,+}\right)$-invariants vanish at all $j \in\{0, \ldots, p-2\}$. For those integers $n \gg 1$, the $p$-adic valuation of the ratio of motivic to automorphic periods over $K_{n}^{+}$is given by

$$
\begin{aligned}
\mu_{p, n}^{\mathrm{Per}}(E)= & n\left((p-1) \cdot p^{n-1}-\lambda_{E}^{\mathrm{cy}}+1\right) \\
& -\left(p^{n-1}+\nabla_{K_{n}^{+}}^{\mathrm{Sym}^{2} E}+\operatorname{ord}_{p}\left(h^{-}\left(K_{n}\right)\right)\right)+O(1),
\end{aligned}
$$

where $\nabla_{K_{n}^{+}}^{\mathrm{Sym}^{2} E}$ denotes the p-adic order of

$$
\begin{aligned}
\prod_{\substack{\text { places } \nu \mid N_{E} \\
\text { of } K_{n}^{+}}} \operatorname{det}(1 & -\left(N_{K_{n}^{+} / \mathbb{Q}}(\nu)\right)^{-s} \\
& \left.\times \operatorname{Frob}_{\nu} \mid\left(\operatorname{Sym}^{2} H_{p}^{1}(E)\right)^{I_{\nu}}\right)\left.\right|_{s=2} .
\end{aligned}
$$

It was rather surprising to see these bad Euler factors for the symmetric square $L$-function playing such a crucial role in the final formula (an algorithm outlining how to compute these error terms $\nabla_{K_{n}^{+}}^{\mathrm{Sym}^{2} E}$ is supplied as an appendix to this paper; see Section 6). The demonstration of this result uses a version of the two-variable main 
conjecture proven by Karl Rubin in the case of complex multiplication.

Lastly let us recall from [Hida and Tilouine 93, Section 0] that the anticyclotomic main conjecture over $K_{n}^{+}$ affirms that $\mathbb{H}_{\lambda}=($ a unit $) \times h^{-}\left(K_{n}\right) \times \mathbb{L}_{\left(K_{n}, \lambda\right)}^{-}$, where $\mathbb{L}_{\left(K_{n}, \lambda\right)}^{-}$denotes the branch of the Katz $p$-adic $L$-function projected along $\left(K_{n}^{+}\right)^{\text {anti }}$. We therefore have the following consequence.

Corollary 1.9. For all integers $n \gg 1$,

$$
\begin{aligned}
\operatorname{ord}_{p} & \left(\frac{\mathcal{L}^{\mathrm{Hida}}\left(E, \rho_{n}\right)}{\mathcal{L}\left(E, \rho_{n}\right)}\right) \\
= & n\left((p-1) \cdot p^{n-1}-\lambda_{E}^{\mathrm{cy}}+1\right)-\left(p^{n-1}+\nabla_{K_{n}^{+}}^{\mathrm{Sym}^{2} E}\right) \\
& +\operatorname{ord}_{p}\left(\mathbb{L}_{\left(K_{n}, \lambda\right)}^{-}(2, \ldots, 2)\right)+\text { a fixed constant. }
\end{aligned}
$$

\section{THETA LIFTS AND THE KATZ MEASURE}

Let $M$ be a CM field, and $M^{+}$its maximal real subfield. As usual, one fixes embeddings $\iota_{\infty}: \overline{\mathbb{Q}} \hookrightarrow \mathbb{C}$ and $\iota_{p}: \overline{\mathbb{Q}} \hookrightarrow \mathbb{C}_{p}$, and writes $c \in \operatorname{Aut}(\overline{\mathbb{Q}})$ for complex conjugation. We shall also assume that every prime of $M^{+}$ above $p$ splits inside $M$.

In particular, this permits us to pick a $p$-ordinary $\mathrm{CM}$ type for $M$, i.e., one can choose a set $\Sigma$ of embeddings of $M$ into $\overline{\mathbb{Q}}$ such that

(i) $\Sigma \cap \Sigma \circ c=\varnothing$, and $\Sigma \cup \Sigma \circ c$ is the set of all embeddings of $M$ into $\overline{\mathbb{Q}}$;

(ii) the $p$-adic place induced by $\sigma \in \Sigma$ composed with $\iota_{p}$ is distinct from that induced by an element $\sigma^{\prime} \in$ $\Sigma \circ c$.

A good reference for the theory of theta lifts is contained in the article [Hida and Tilouine 93, Sections 68]. Following that exposition, $\mathfrak{G}_{\infty}(\mathfrak{C})$ indicates the Galois group of $M\left(\mathfrak{C} p^{\infty}\right)$ over $M$, where $M\left(\mathfrak{C} p^{\infty}\right)$ is the maximal ray class field modulo $\mathfrak{C} p^{\infty}$. There is a decomposition

$$
\mathfrak{G}_{\infty}(\mathfrak{C})=\mathfrak{G}_{\infty}(\mathfrak{C})_{\text {tors }} \times \mathbf{W}, \quad \zeta . w \leftrightarrow(\zeta, w),
$$

and the free part $\mathbf{W}=\mathbf{W}_{M}$ is determined independently of the tame conductor $\mathfrak{C}$.

Put $N=\mathfrak{C} \times \mathfrak{C}^{c} \times D_{M / M^{+}}$, with $D_{M / M^{+}}$indicating the relative discriminant. The theta measure [Hida and Tilouine 93, Section 6] is a canonical morphism

$$
\theta_{N}^{*}: \mathbf{h}^{\text {n.ord }}\left(N ; \mathcal{O}_{\mathbb{C}_{p}}\right) \longrightarrow \mathcal{O}_{\mathbb{C}_{p}}\left[\left[\mathfrak{G}_{\infty}(\mathfrak{C})\right]\right]
$$

of the nearly ordinary Hecke algebra of level $N$ into the completed group ring. Furthermore, given a multiplicative character $\eta: \mathfrak{G}_{\infty}(\mathfrak{C})_{\text {tors }} \rightarrow \mathcal{O}_{\mathbb{C}_{p}}^{\times}$, there is an obvious projection $\eta_{*}: \mathcal{O}_{\mathbb{C}_{p}} \llbracket \mathfrak{G}_{\infty}(\mathfrak{C}) \rrbracket \rightarrow \Lambda_{0}:=\mathcal{O}_{\mathbb{C}_{p}} \llbracket \mathbf{W} \rrbracket$.

Notation 2.1. Write $C_{0}(\lambda)$ for the congruence module associated to the composition $\lambda=\eta_{*} \circ \theta_{N}^{*}$ : $\mathbf{h}^{\text {n.ord }}\left(N ; \mathcal{O}_{\mathbb{C}_{p}}\right) \rightarrow \Lambda_{0}$; in the terminology of [Hida and Tilouine 93, Section 6.9],

$C_{0}(\lambda):=(\mathcal{R}(\mathbf{K}) \oplus \mathcal{R}(\mathbf{B})) / \mathcal{R} \cong \mathcal{R}(\mathbf{K}) /\{\mathcal{R} \cap(\mathcal{R}(\mathbf{K}) \oplus 0)\}$,

where $\mathbf{h}\left(\eta^{+}, \eta^{\prime}\right)=\mathbf{K} \oplus \mathbf{B}$ with $\mathbf{K}=\operatorname{Frac}\left(\Lambda_{0}\right)$, and $\left(\eta^{\prime}, \eta^{+}\right)$ is the character of $\lambda$.

Let $\mathbb{H}_{\lambda} \in \mathcal{O}_{\mathbb{C}_{p}} \llbracket \mathbf{W} \rrbracket$ denote the characteristic power series of $C_{0}(\lambda)$; in other words, the element $\mathbb{H}_{\lambda}$ generates the smallest principal ideal containing $\mathcal{R} \cap(\mathcal{R}(\mathbf{K}) \oplus 0)$. The following is quite deep.

Theorem 2.2. [Mazur and Tilouine 90, Tilouine 89, Hida and Tilouine 93] Under the condition $\mathfrak{C}+\mathfrak{C}^{c}=\mathcal{O}_{M}$, up to units,

$$
\mathbb{H}_{\lambda}=\frac{\# \operatorname{Pic}\left(\mathcal{O}_{M}\right)}{\# \operatorname{Pic}\left(\mathcal{O}_{M^{+}}\right)} \times \mathbb{L}_{(M, \lambda)}^{-},
$$

where the branch $\mathbb{L}_{(M, \lambda)}^{-}=\pi_{\eta}^{-}\left(\mu^{\mathrm{Katz}}\right)$ is projected along $\pi_{\eta}^{-}: \mathcal{O}_{\mathbb{C}_{p}} \llbracket \mathfrak{G}_{\infty}\left(\mathfrak{C}^{-}\right) \rrbracket \rightarrow \Lambda_{0}$ via $(\zeta, w) \mapsto \eta^{-1}(\eta \circ c)(\zeta) \times$ $w^{-1} w^{c}$.

This result forms a significant component of the "anticyclotomic main conjecture." In the context of this paper, the above provides us with a computational method of determining the valuation of $\mathbb{H}_{\lambda}$ at weight two (i.e., for CM elliptic curves).

We now outline the precise role $C_{0}(\lambda)$ plays in the nonabelian Iwasawa theory. Recall that $\lambda$ : $\mathbf{h}^{\text {n.ord }}\left(N ; \mathcal{O}_{\mathbb{C}_{p}}\right) \longrightarrow \Lambda_{0}$ was the mapping induced at level $N$ by $\eta$. We now consider a secondary mapping

$$
\nu=\gamma_{*} \circ \theta_{N^{\prime}}^{*}: \mathbf{h}^{\text {n.ord }}\left(N^{\prime} ; \mathcal{O}_{\mathbb{C}_{p}}\right) \longrightarrow \Lambda_{0}
$$

defined in an analogous fashion (note that the levels $N$ and $N^{\prime}$ need not be coprime).

Theorem 2.3. [Hida 91] There exists an element $\mathfrak{D}_{\lambda, \nu} \in$ $\operatorname{Frac}\left(\Lambda_{0} \widehat{\otimes}_{\mathcal{O}_{\mathbb{C}_{p}}} \Lambda_{0}\right)$ satisfying:

(a) $\left(\mathbb{H}_{\lambda} \otimes 1\right) \cdot \mathfrak{D}_{\lambda, \nu} \in \Lambda_{0} \widehat{\otimes}_{\mathcal{O}_{\mathbb{C}_{p}}} \Lambda_{0}$.

(b) For all critical motivic points

$$
(\mathcal{P}, \mathcal{Q}) \in \operatorname{Spec}\left(\Lambda_{0} \widehat{\otimes}_{\mathcal{O}_{\mathbb{C}_{p}}} \Lambda_{0}\right)\left(\overline{\mathbb{Q}}_{p}\right),
$$


we have

$$
\begin{aligned}
\mathfrak{D}_{\lambda, \nu}(\mathcal{P}, \mathcal{Q})= & \frac{C(\mathcal{P}, \mathcal{Q}) W(\mathcal{P}, \mathcal{Q}) E(\mathcal{P}, \mathcal{Q})}{S(\mathcal{P})} \\
& \times \frac{L_{p}\left(\theta\left(\lambda_{\mathcal{P}}\right) \otimes \theta\left(\nu_{\mathcal{Q}}\right)^{\vee}, 0\right)}{\left\langle\theta\left(\lambda_{\mathcal{P}}\right) \otimes \eta_{\mathcal{P}}^{\prime}, \theta\left(\lambda_{\mathcal{P}}\right) \otimes \eta_{\mathcal{P}}^{\prime}\right\rangle},
\end{aligned}
$$

where $\lambda_{\mathcal{P}}=\mathcal{P} \circ \lambda$ and $\nu_{\mathcal{Q}}=\mathcal{Q} \circ \nu$.

We need to explain some of the notation used here. The primitive Hilbert modular forms $\theta\left(\lambda_{\mathcal{P}}\right)$ and $\theta\left(\nu_{\mathcal{Q}}\right)$ over $M^{+}$are theta lifts, corresponding to the Hecke characters $\lambda_{\mathcal{P}}=\mathcal{P} \circ \lambda$ and $\nu_{\mathcal{Q}}=\mathcal{Q} \circ \nu$ respectively. The $L$-function itself is the Rankin convolution of $\theta\left(\lambda_{\mathcal{P}}\right)$ with the dual of $\theta\left(\nu_{\mathcal{Q}}\right)$. Denoting by $-^{u}$ the unitarization, then on the level of formal Dirichlet series,

$$
L_{p}\left(\theta\left(\lambda_{\mathcal{P}}\right) \otimes \theta\left(\nu_{\mathcal{Q}}\right)^{\vee}, s-1+(m(\mathcal{P})-m(\mathcal{Q})) / 2\right)
$$

equals $L_{p}\left(\left(\lambda_{\mathcal{P}}^{u} \nu_{\mathcal{Q}}^{c u}\right)^{\text {prim }}, s\right) \cdot L_{p}\left(\left(\lambda_{\mathcal{P}}^{u} \nu_{\mathcal{Q}}\left(-^{c}\right)^{c u}\right)^{\text {prim }}, s\right)$, up to some bad Euler factors.

Lastly, the denominator in Theorem 2.3(b) is the automorphic period associated to the Petersson self-product of $\theta\left(\lambda_{\mathcal{P}}\right) \otimes \eta_{\mathcal{P}}^{\prime}$.

Remark 2.4. The interpolation factors are quite ugly, and are given in [Hida 91, p. 317]. Suffice to say that for a motivic pair $\left(\lambda_{\mathcal{P}}, \nu_{\mathcal{Q}}\right)$ as above, all the $\epsilon$-factors $W(\mathcal{P}, \mathcal{Q})$, the $\Gamma$-factors $C(\mathcal{P}, \mathcal{Q})$, and Euler factors $E(\mathcal{P}, \mathcal{Q}) S(\mathcal{P})^{-1}$ coincide exactly with those in the general recipe of [Coates and Perrin-Riou 89, Section 4.14] (this is checked meticulously by Hida in the preamble to his article).

\subsection{A Special Case: CM Elliptic Curves}

Henceforth we shall confine ourselves exclusively to the situation of the introduction. We take

$$
M=K_{n}=\mathbb{Q}\left(\sqrt{-D}, \mu_{p^{n}}\right)
$$

as our CM field, and consider the setup of Figure 1. The trick now is to make a judicious choice of $\lambda_{\mathcal{P}}$ and $\nu_{\mathcal{Q}}$.

Let $\mathfrak{C}=\operatorname{cond}_{K_{n}}(E) \subset \mathcal{O}_{K_{n}}$, and choose $\lambda$ : $\mathbf{h}^{\text {n.ord }}\left(N ; \mathcal{O}_{\mathbb{C}_{p}}\right) \rightarrow \Lambda_{0}$ to be the homomorphism that corresponds (by duality) to the $p$-ordinary Hida family, lifting the base-change of $f_{E} \in \mathcal{S}_{2}\left(\Gamma_{0}\left(N_{E}\right)\right)$ over $K_{n}^{+}$. Further, let us take $\mathcal{P}: \Lambda_{0} \rightarrow \mathbb{C}_{p}$ to be the specialization at weight $\underline{k}=(2, \ldots, 2)$.

Then the Hilbert modular form $\theta\left(\lambda_{\mathcal{P}}\right)$ is tautologically the base-change of $f_{E}$, and its complex $L$-series coincides with $L\left(\left(\Phi_{E / K_{n}}\right)^{u}, s-1 / 2\right)$, where $\Phi_{E / K}$ is the Grössencharakter attached to $E$.
On the other hand, initially we fix a character $\psi$ : $U^{(n)} \rightarrow \overline{\mathbb{Q}}_{p}^{\times}$of finite order. For $j$ in the range $\{0, \ldots, n\}$, one recalls that

$$
\chi_{j}: \operatorname{Gal}\left(K_{j}\left(p^{j} \sqrt{\Delta}\right) / K_{j}\right) \longrightarrow \mu_{p^{j}}
$$

was the anticyclotomic character inducing the Artin representation " $\rho_{j}$ " over the $\mathrm{CM}$ field $K$; in particular, $\chi_{j}: \sigma \mapsto \sigma\left(\sqrt[p^{j}]{\Delta}\right) / \sqrt[p^{j}]{\Delta}$.

Remark 2.5. Suppose that $\mathfrak{C}^{\prime}$ is divisible by the conductor of $\operatorname{Res}_{K_{n}}\left(\chi_{j}\right) \otimes \psi$. Then there is a unique map at level $N^{\prime}=\mathfrak{C}^{\prime} \mathfrak{C}^{\prime c} D_{K_{n} / K_{n}^{+}}$lifting this character, i.e.,

$$
\nu_{\mathcal{Q}}=\nu_{\mathcal{Q}}(j, n, \psi): \mathbf{h}^{\text {n.ord }}\left(N^{\prime} ; \mathcal{O}_{\mathbb{C}_{p}}\right) \stackrel{\gamma_{*} \circ \theta_{N^{\prime}}^{*}}{\longrightarrow} \Lambda_{0} \stackrel{\mathcal{Q}}{\longrightarrow} \mathbb{C}_{p}
$$

where $\gamma=\left.\operatorname{Res}_{K_{n}}\left(\chi_{j}\right) \otimes \psi\right|_{G_{\infty}\left(\mathfrak{C}^{\prime}\right)_{\text {tors }}}$, and $\mathcal{Q}$ denotes the arithmetic specialization to weight $\underline{k}^{\prime}=(1, \ldots, 1)$ with character $\left.\operatorname{Res}_{K_{n}}\left(\chi_{j}\right) \otimes \psi\right|_{\mathbf{W}}$.

Clearly $\left.\nu_{\mathcal{Q}}\right|_{G_{\infty}\left(\mathfrak{C}^{\prime}\right)}$ gives rise to a unitary Hecke character. Moreover, $\theta\left(\nu_{\mathcal{Q}}(j, n, \psi)\right)$ is the primitive Hilbert modular form associated to the Gal $\left(\overline{\mathbb{Q}} / K_{n}^{+}\right)$representation

$$
\operatorname{Ind}_{K_{n}}^{K_{n}^{+}}\left(\operatorname{Res}_{K_{n}}\left(\chi_{j}\right)\right) \otimes \psi
$$

via the fundamental work of Serre.

Question 2.6. What $L$-value data does this pair $\left(\lambda_{\mathcal{P}}, \nu_{\mathcal{Q}}\right)$ interpolate?

Applying standard properties of the theta lift mentioned earlier,

$$
\begin{aligned}
L_{p}( & \left.\theta\left(\lambda_{\mathcal{P}}\right) \otimes \theta\left(\nu_{\mathcal{Q}}\right)^{\vee}, 0\right) \\
= & L_{p}\left(\left(\lambda_{\mathcal{P}}^{u} \nu_{\mathcal{Q}}^{c u}\right)^{\operatorname{prim}}, 1 / 2\right) \cdot L_{p}\left(\left(\lambda_{\mathcal{P}}^{u} \nu_{\mathcal{Q}}\left(-^{c}\right)^{c u}\right)^{\operatorname{prim}}, 1 / 2\right) \\
= & L_{p}\left(\left(\Phi_{E / K_{n}}\right)^{u} \nu_{\mathcal{Q}}^{c}, s-1 / 2\right) \\
\quad & \times\left. L_{p}\left(\left(\Phi_{E / K_{n}}\right)^{u} \nu_{\mathcal{Q}}\left(-{ }^{c}\right)^{c}, s-1 / 2\right)\right|_{s=1} .
\end{aligned}
$$

After unraveling the unitarization, then removing the $\mathrm{Eu}-$ ler factor contribution coming from the $E(\mathcal{P}, \mathcal{Q})$-term, one obtains the special value

$$
\begin{aligned}
& L_{\{p \Delta\}}\left(E / K_{n}, \operatorname{Res}_{K_{n}}\left(\chi_{j}\right)^{c} \otimes \psi^{-1}, 1\right) \\
& \quad=\prod_{\beta: G\left(K_{n} / K_{j}\right) \rightarrow \mathbb{C}^{\times}} L_{\{p \Delta\}}\left(E / K, \rho_{j} \otimes \beta \psi^{-1}, 1\right) .
\end{aligned}
$$




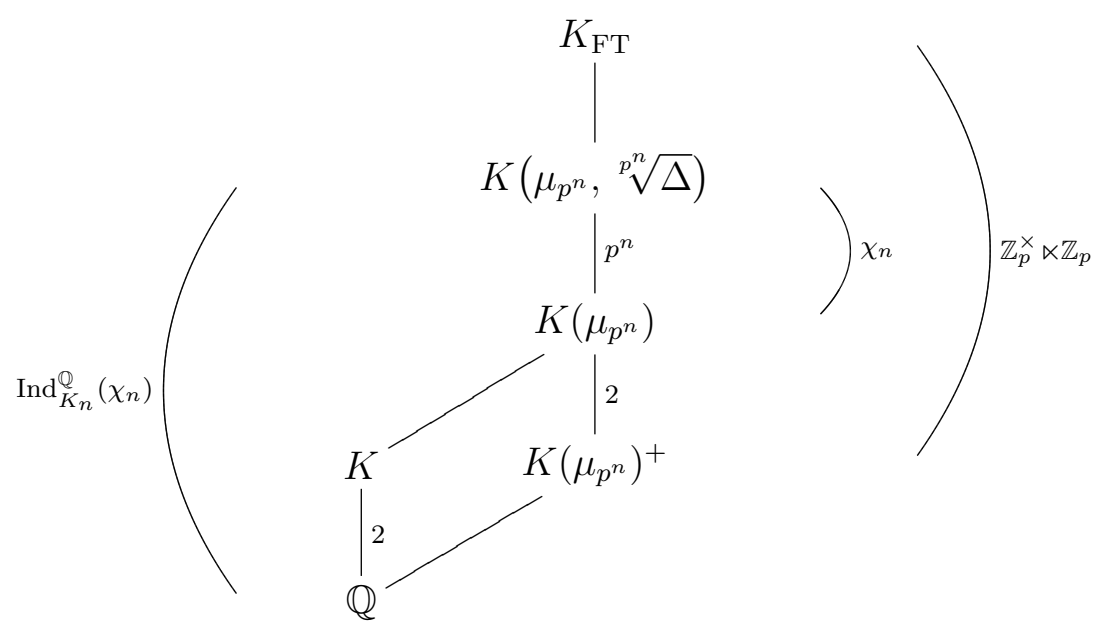

FIGURE 1. Fields in the false Tate tower.

Comparing Definition 1.1 with Theorem 2.3, it can readily be seen that $\left(\mathbb{H}_{\lambda}(\mathcal{P}) \otimes 1\right) \cdot \mathfrak{D}_{\lambda, \nu}(\mathcal{P}, \mathcal{Q})$ equals

$$
\begin{aligned}
& \frac{\left(\pi^{-2} \Omega_{E}^{+} \Omega_{E}^{-}\right)^{\left[K_{n}^{+}: \mathbb{Q}\right]}}{\left\langle\theta\left(\lambda_{\mathcal{P}}\right) \otimes \eta_{\mathcal{P}}^{\prime}, \theta\left(\lambda_{\mathcal{P}}\right) \otimes \eta_{\mathcal{P}}^{\prime}\right\rangle} \times \mathbb{H}_{\lambda}(\mathcal{P}) \\
& \quad \times \psi\left(\operatorname{Norm}_{j, n}\left(\mathcal{L}\left(E, \rho_{j}\right)\right)\right) .
\end{aligned}
$$

The first two terms in the product are nonzero scalars; in particular, they do not depend on how the character $\psi$ is chosen, nor on the Artin representation $\rho_{j}$. Estimating the powers of $p$ occurring in these scalars will occupy a substantial portion of the remainder of this article.

Definition 2.7. One denotes the automorphy defect at weight $(2, \ldots, 2)$ by

$$
\operatorname{Err}_{K_{n}^{+}}(E):=\frac{\left(\pi^{-2} \Omega_{E}^{+} \Omega_{E}^{-}\right)^{\left[K_{n}^{+}: \mathbb{Q}\right]}}{\left\langle\theta\left(\lambda_{\mathcal{P}}\right) \otimes \eta_{\mathcal{P}}^{\prime}, \theta\left(\lambda_{\mathcal{P}}\right) \otimes \eta_{\mathcal{P}}^{\prime}\right\rangle} \times\left|\mathbb{H}_{\lambda}(\mathcal{P})\right|_{p}^{-1},
$$

which is a well-defined scalar, depending on both $E$ and the field $K_{n}^{+}=K_{n} \cap \mathbb{R}$.

Essentially, this error term measures the ratio of the "automorphic" $p$-adic $L$-function

$$
\left.\left(\mathbb{H}_{\lambda}(\mathcal{P}) \otimes 1\right) \cdot \mathfrak{D}_{\lambda, \nu}(\mathcal{P},-)\right|_{\mathcal{O}_{\mathbb{C}_{p}} \llbracket U^{(n)} \rrbracket}
$$

to its motivic counterpart $\operatorname{Norm}_{j, n}\left(\mathcal{L}\left(E, \rho_{j}\right)\right)$. If $\mathrm{Hy}$ pothesis 1.2 holds for the given $n$, then the norm of the motivic $p$-adic $L$-function is $p$-integral.
We should point out that the family of automorphic $L$-functions

$$
\left.\mathfrak{D}_{\lambda, \nu}(\mathcal{P},-)\right|_{\mathcal{O}_{\mathbb{C}_{p}} \llbracket U^{(n)} \rrbracket}
$$

associated to

$$
\gamma=\left.\operatorname{Res}_{K_{n}}\left(\chi_{j}\right) \otimes \psi\right|_{G_{\infty}\left(\mathfrak{C}^{\prime}\right)_{\text {tors }}}
$$

share a common $\mu$-invariant, for all $j \in\{0, \ldots, n\}$ and fixed character $\left.\psi\right|_{G_{\infty}\left(\mathfrak{C}^{\prime}\right)_{\text {tors }}}$ (since switching $\gamma^{\prime}$ 's does not change the branch of the underlying ray class measure).

Moreover, under Hypothesis 1.3, the $\mu$-invariant of $\operatorname{Norm}_{0,1}\left(\mathcal{L}\left(E, \rho_{0}\right)\right)$ is trivial; hence the $\mu$-invariant of $\operatorname{Norm}_{0, n}\left(\mathcal{L}\left(E, \rho_{0}\right)\right)$ must also vanish for every $n \geq 1$. It follows that the quantity $\operatorname{ord}_{p}\left(\mathbf{E r r}_{K_{n}^{+}}(E)\right)$ coincides with the $\mu$-invariant of $\left.\mathfrak{D}_{\lambda, \nu}(\mathcal{P},-)\right|_{\mathcal{O}_{\mathbb{C}_{p}} \llbracket U^{(n)} \rrbracket}$ for the particular character choice $\gamma=\left.\operatorname{Res}_{K_{n}}(\mathbf{1}) \otimes \psi\right|_{G_{\infty}\left(\mathfrak{C}^{\prime}\right)_{\text {tors }}}$. Finally (cf. the previous paragraph), this quantity will be the common $\mu$-invariant shared by the family of automorphic $p$-adic $L$-functions that we have been considering.

Key Fact 2.8. Because $\operatorname{Res}_{K_{n}}\left(\chi_{j}\right)$ takes values in $\mu_{p^{\infty}}$, such characters are congruent to 1 modulo " $\mathfrak{M}_{\mathbb{C}_{p}}$," the maximal ideal of $\mathcal{O}_{\mathbb{C}_{p}}$. Thus each residual specialization as in Figure 2, while dependent on $n$ and $\psi$, is independent of the choice of $j \in\{0, \ldots, n\}$. 


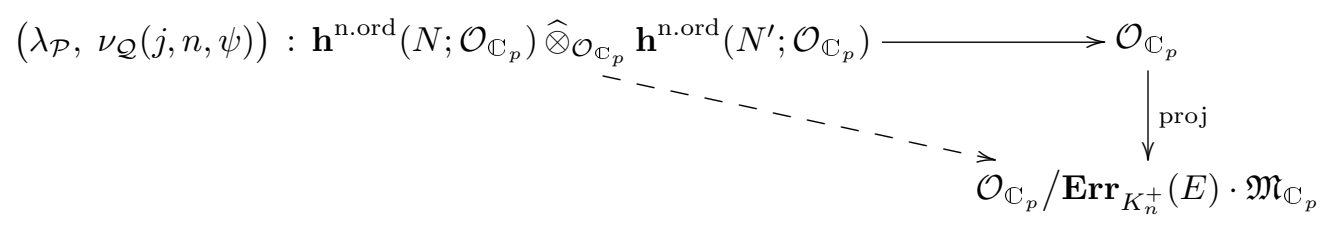

FIGURE 2. Residual specialization of the Hecke algebras.

As a corollary, clearly $\left(\mathbb{H}_{\lambda}(\mathcal{P}) \otimes 1\right) \cdot \mathfrak{D}_{\lambda, \nu}(\mathcal{P}, \mathcal{Q})$ modulo $\mathbf{E r r}_{K_{n}^{+}}(E) \cdot \mathfrak{M}_{\mathbb{C}_{p}}$ must also be independent of the choice of $j \in\{0, \ldots, n\}$. Allowing $\psi$ to range over all finite-order characters of $U^{(n)}$, equivalently $\left(\mathbb{H}_{\lambda}(\mathcal{P}) \otimes 1\right) \cdot \mathfrak{D}_{\lambda, \nu}(\mathcal{P},-)$ $\bmod \mathbf{E r r}_{K_{n}^{+}}(E) \cdot \mathfrak{M}_{\mathbb{C}_{p}} \llbracket U^{(n)} \rrbracket$ is independent of $j$, whence

$$
\begin{aligned}
\mathcal{L}\left(E, \rho_{n}\right) & \equiv \operatorname{Norm}_{n-1, n}\left(\mathcal{L}\left(E, \rho_{n-1}\right)\right) \equiv \cdots \\
& \equiv \operatorname{Norm}_{0, n}\left(\mathcal{L}\left(E, \rho_{0}\right)\right) \quad \bmod \mathfrak{M}_{\mathbb{C}_{p}} \llbracket U^{(n)} \rrbracket,
\end{aligned}
$$

which is very close to what is actually required.

Remark 2.9. It is shown in [Bouganis and Dokchitser 07, Theorem 4.2] that the algebraic part of the $L$-value of $E$, twisted by the Artin representation $\rho$, lies in the field of definition of $E$ and $\rho$. Given that the self-dual representations $\operatorname{Ind}_{K}^{\mathbb{Q}}\left(\rho_{n}\right)$ are all realizable over the rational numbers (as is the curve $E$ ), the above strengthens to yield a congruence modulo $\mathfrak{M}_{\mathbb{C}_{p}} \llbracket U^{(n)} \rrbracket \cap \mathbb{Q}_{p} \llbracket U^{(n)} \rrbracket$. However, this latter ring is none other than $p \cdot \mathbb{Z}_{p} \llbracket U^{(n)} \rrbracket$, which completes the proof of Theorem 1.4.

\section{CALCULATING MOTIVIC VERSUS AUTOMORPHIC PERIODS}

We will now describe the numerical ratio of $\mathcal{L}\left(E, \rho_{n}\right)$ to its automorphic counterpart. Under the conditions of Theorem 1.4, this ratio corresponds to the $\mu$-invariant of $\left(\mathbb{H}_{\lambda}(\mathcal{P}) \otimes 1\right) \cdot \mathfrak{D}_{\lambda, \nu}(\mathcal{P},-)$ precisely. From Definition 2.7, it can be written as

$$
\operatorname{ord}_{p}\left(\operatorname{Err}_{K_{n}^{+}}(E)\right)=\mu_{p, n}^{\mathrm{anti}}(E)+\mu_{p, n}^{\mathrm{Per}}(E),
$$

where

$$
\begin{aligned}
\mu_{p, n}^{\operatorname{anti}}(E)= & \operatorname{ord}_{p}\left(h\left(K_{n}\right)\right)-\operatorname{ord}_{p}\left(h\left(K_{n}^{+}\right)\right) \\
& +\operatorname{ord}_{p}\left(\mathbb{L}_{\left(K_{n}, \lambda\right)}^{-}(\mathcal{P})\right)
\end{aligned}
$$

by Theorem 2.2 , and secondly,

$$
\begin{aligned}
\mu_{p, n}^{\mathrm{Per}}(E)=\operatorname{ord}_{p}( & \left(\pi^{-2} \Omega_{E}^{+} \Omega_{E}^{-}\right)^{\left[K_{n}^{+}: \mathbb{Q}\right]} \\
& \left.\times\left\langle\theta\left(\lambda_{\mathcal{P}}\right) \otimes \eta_{\mathcal{P}}^{\prime}, \theta\left(\lambda_{\mathcal{P}}\right) \otimes \eta_{\mathcal{P}}^{\prime}\right\rangle^{-1}\right) .
\end{aligned}
$$

Theorem 3.1. (Katz.) In the notation of [Katz 78, Sections 5.7.8-5.7.9], $\mathbb{L}_{\left(K_{n}, \lambda\right)}^{-}(\mathcal{P})$ equals

$$
\begin{aligned}
& \Omega_{p}^{m_{0} \Sigma+2 d} \times\left[\mathcal{O}_{K_{n}}^{\times}: \mathcal{O}_{K_{n}^{+}}^{\times}\right] \cdot W_{p}\left((\lambda \circ c) \cdot \lambda^{-1}\right) \\
& \times \frac{(-1)^{m_{0} \Sigma}}{\sqrt{\operatorname{Disc}\left(K_{n}^{+}\right)}} \times \frac{L_{\mathfrak{B}, \mathfrak{B}^{*}}\left((\lambda \circ c)_{\mathcal{P}} \cdot \lambda_{\mathcal{P}}^{-1}, 0\right)}{\pi^{-d} \times \Omega_{\infty}^{m_{0} \Sigma+2 d}},
\end{aligned}
$$

where $\lambda_{\mathcal{P}}=\left(\Phi_{E / K_{n}}\right)^{u}, W_{p}$ is a root number, and $\left(\Omega_{\infty}, \Omega_{p}\right)$ denote the Katz periods.

From a computational perspective, these terms are straightforward to work out. For example, the index $\left[\begin{array}{lll}\mathcal{O}_{K_{n}}^{\times} & : & \mathcal{O}_{K_{n}^{+}}^{\times}\end{array}\right]$is just a power of $p$, while the pe$\operatorname{riod} \Omega_{p}$ is always a $p$-adic unit. Finally, the ratio $L_{\mathfrak{B}, \mathfrak{B}^{*}}(-) / \Omega_{\infty}^{m_{0} \Sigma+2 d}$ can be calculated as the quotient of a Hecke $L$-series and the value of an Eisenstein-Damerell series at 0 .

Question 3.2. How does one calculate the quantity $\mu_{p, n}^{\mathrm{Per}}(E)$ numerically?

For any Dirichlet character $\psi$ whose conductor is coprime to that of $E$, we will write $L^{\mathrm{imp}}\left(\operatorname{Sym}^{2} E, \psi, s\right)$ for the imprimitive $\psi$-twisted symmetric square $L$ series. Normalizing the choice of Gauss sum by $\tau(\psi)=$ $\sum_{a=1}^{\mathfrak{f}_{\psi}} \psi(a) e^{2 \pi i a / \mathfrak{f}_{\psi}}$, one may define

$$
\xi\left(E / K_{n}^{+}\right) \quad:=\prod_{\psi: G\left(K_{n}^{+} / \mathbb{Q}\right) \rightarrow \mathbb{C}^{\times}} \frac{\tau\left(\psi^{-2}\right) L^{\mathrm{imp}}\left(\mathrm{Sym}^{2} E, \psi, 2\right)}{\pi^{3}\left\langle f_{E}, f_{E}\right\rangle_{N_{E}}},
$$

which is $\operatorname{Aut}(\mathbb{C})$-invariant (and hence rational) due to results in [Sturm 80, Sturm 89].

Proposition 3.3. If the prime $p \neq 2$ does not divide $\operatorname{deg}\left(X_{0}\left(N_{E}\right) \stackrel{\varphi_{E}}{\rightarrow} E\right)$, then

$$
\begin{aligned}
& \frac{\left\langle\theta\left(\lambda_{\mathcal{P}}\right) \otimes \eta_{\mathcal{P}}^{\prime}, \theta\left(\lambda_{\mathcal{P}}\right) \otimes \eta_{\mathcal{P}}^{\prime}\right\rangle}{\left(\pi^{-2} \Omega_{E}^{+} \Omega_{E}^{-}\right)^{\left[K_{n}^{+}: \mathbb{Q}\right]}} \\
& \quad=(\text { p-adic unit }) \times \frac{\left|\operatorname{Disc}\left(K_{n}^{+}\right)\right|_{\infty}}{\left|\operatorname{Disc}\left(\mathbb{Q}\left(\mu_{p^{n}}\right)^{+}\right)\right|_{\infty}} \times \xi\left(E / K_{n}^{+}\right) .
\end{aligned}
$$


The demonstration of this result is given at the end of this section.

It is a basic exercise in the arithmetic of cyclotomic fields to show that

$$
\left|\operatorname{Disc}\left(K_{n}^{+}\right)\right|_{p}^{-1}=p^{p^{n-1}(p n-n-1)}
$$

and also

$$
\left|\operatorname{Disc}\left(\mathbb{Q}\left(\mu_{p^{n}}\right)^{+}\right)\right|_{p}^{-1}=p^{\left(p^{n-1}(p n-n-1)-1\right) / 2} .
$$

The calculation of the period ratio therefore reduces to a computation of $\psi$-twisted symmetric square $L$-series at $s=2$. Using the computer package MAGMA and the subroutine LSeries, we computed the $L$-values to very high precision and obtained $\xi\left(E / K_{n}^{+}\right)$as a rational number.

Due to irksome computational issues, from now on we restrict ourselves to the layer $n=1$ exclusively. Let us focus on just the first few CM elliptic curves over $\mathbb{Q}$ of rank zero and minimal conductor, namely the curves $27 A(1), 32 A(1)$, and $49 A(1)$.

Remark 3.4. We also (temporarily) suppose that the analytic rank of $E / K\left(\mu_{p}\right)$ is zero. For any discrete $p$-primary $G_{\infty}$-module $\mathcal{S}$, one may define

$$
\mathbf{E C}\left(G_{\infty}, \mathcal{S}\right):=\prod_{j=0}^{\infty} \#\left(H^{j}\left(G_{\infty}, \mathcal{S}\right)\right)^{(-1)^{j}}
$$

assuming that these cohomologies are finite.

It is shown in [Hachimori and Venjakob 03, Proposition 4.12] that the classical $p$-primary Selmer group of $E$ over the $p$-adic Lie extension $K_{\mathrm{FT}}$ is trivial if and only if one has the numerical condition $\mathbf{E C}\left(G_{\infty}, \operatorname{Sel}_{K_{\mathrm{FT}}}(E)_{p^{\infty}}\right)=1$. Moreover, the latter Euler characteristic will coincide with the leading term of $\mathcal{L}_{p}\left(E, \rho_{0}\right)$ (up to a unit), provided that the full BirchSwinnerton-Dyer conjecture holds for $E$ over $K\left(\mu_{p}\right)$.

To compute $\mathbf{1}\left(\mathcal{L}_{p}\left(E, \rho_{0}\right)\right)$ and check whether it is a $p$-adic unit, it is enough to calculate the quantity $\mathcal{X}_{E}\left(\operatorname{Reg}_{\mathbb{Q}\left(\mu_{p}\right) / \mathbb{Q}}\right)$. Note that here for a general Artin representation $\rho: G_{\infty} \rightarrow \mathrm{GL}(V)$, we write

$$
\begin{aligned}
\mathcal{X}_{E}(\rho):= & \epsilon_{p}(\rho) \times \frac{P_{p}\left(\rho^{\vee}, \alpha_{p}^{-1}\right)}{P_{p}\left(\rho, p^{-1} \alpha_{p}\right)} \times \alpha_{p}^{-\mathfrak{f}_{p}(\rho)} \\
& \times \frac{L_{\{p \Delta\}}(E, \rho, 1)}{\left(\Omega_{E}^{+}\right)^{\operatorname{dim}^{+} \rho}\left(\Omega_{E}^{-}\right)^{\operatorname{dim}^{-} \rho}} .
\end{aligned}
$$

This follows because

$$
\begin{aligned}
\mathbf{1}\left(\mathcal{L}_{p}\left(E, \rho_{0}\right)\right) & =\mathcal{X}_{E}\left(\operatorname{Reg}_{\mathbb{Q}\left(\sqrt{-D}, \mu_{p}\right) / \mathbb{Q}}\right) \\
& =\mathcal{X}_{E}\left(\operatorname{Reg}_{\mathbb{Q}\left(\mu_{p}\right) / \mathbb{Q}}\right)^{2}
\end{aligned}
$$

since the quadratic twist $E \otimes\left(\frac{-D}{}\right)$ is always $\mathbb{Q}$-isogenous to the original elliptic curve.

Bearing in mind our formula for $\mu_{p, n}^{\mathrm{Per}}(E)$ given in Theorem 1.8 when $n \gg 1$, we make the following definition.

Definition 3.5. For each triple $(E, p, \Delta)$, as before, we introduce the quantity

$$
\mu_{p, 1}^{\text {naive }}(E):=(p-1)-\nabla_{K_{1}^{+}}^{\mathrm{Sym}^{2} E}-\operatorname{ord}_{p}\left(h^{-}\left(K_{1}\right)\right),
$$

which is a naive estimate (guess!) of the value $\mu_{p, n}^{\mathrm{Per}}(E)$ at the bottom layer $n=1$.

In Tables 1,2 , and 3 we have tabulated values of $\mu_{p, 1}$ for our three sample elliptic curves. Whenever $\mu_{p, 1}^{\text {Per }}$ and $\mu_{p, 1}^{\text {naive }}$ differ in value, we have highlighted the latter in boldface. After considerable head-scratching, we verified numerically that in all cases our naive guess coincides with the true value if and only if the Katz-Yager $L$-values

$$
\mathbb{L}_{p, K}^{\operatorname{Katz}}\left(\Phi_{E, \mathfrak{p}}^{2} \times \omega_{K}^{j}\right)
$$

are simultaneously $p$-units for all $j \in\{0, \ldots, p-2\}$. Of course, if this condition does not hold, then one expects nontriviality of either the $\mu_{E}^{\mathrm{cy}}$ - or $\lambda_{E}^{\mathrm{cy}}$-invariants, which is bound to alter the final formula somewhat.

Conjecture 3.6. Under this p-unit condition on the constant terms of $\mathbb{L}_{p, K}^{\mathrm{Katz}}$,

$\mu_{p, 1}^{\mathrm{Per}}(E) \stackrel{? ?}{=} \mu_{p, 1}^{\text {naive }}(E):=(p-1)-\nabla_{K_{1}^{+}}^{\mathrm{Sym}^{2} E}-\operatorname{ord}_{p}\left(h^{-}\left(K_{1}\right)\right)$

otherwise, without this condition the equal sign becomes instead a strict less-than inequality.

Certainly this prediction is true in all the examples we have calculated, and we are optimistic that it holds more generally. If it does indeed hold, then it is tantamount to saying that the $O(1)$-term occurring in Theorem 1.8 is precisely the constant zero.

of Proposition 3.3. Our starting point is the beautiful formula [Hida and Tilouine 93, Section 7]:

$$
\begin{aligned}
\left\langle\theta\left(\lambda_{\mathcal{P}}\right) \otimes \eta_{\mathcal{P}}^{\prime}, \theta\left(\lambda_{\mathcal{P}}\right) \otimes \eta_{\mathcal{P}}^{\prime}\right\rangle_{N} \\
=\left|\operatorname{Disc}\left(K_{n}^{+}\right)\right|_{\infty} \times \operatorname{Norm}_{K_{n}^{+} / \mathbb{Q}}(N) \times 2^{-2\{2\}+1} \\
\quad \times \pi^{-\left[K_{n}^{+}: \mathbb{Q}\right]-\{2\}} \times L^{\operatorname{imp}}\left(\operatorname{Ad}\left(\theta\left(\lambda_{\mathcal{P}}\right) \otimes \eta_{\mathcal{P}}^{\prime}\right), 1\right)
\end{aligned}
$$

where $N=\mathfrak{C} \times \mathfrak{C}^{c} \times D_{K_{n} / K_{n}^{+}}$was the level of $\theta\left(\lambda_{\mathcal{P}}\right) \otimes$ $\eta_{\mathcal{P}}^{\prime}$. The adjoint $L$-function can be identified with that 


\begin{tabular}{|c|c|c|c|c|c|}
\hline $\boldsymbol{p}$ & $\boldsymbol{\Delta}$ & $\boldsymbol{\mathcal { X }}_{\boldsymbol{E}}\left(\mathbf{R e g}_{\mathbb{Q}\left(\boldsymbol{\mu}_{\boldsymbol{p}}\right) / \mathbb{Q}}\right)$ & $\mathbf{S e l}_{\boldsymbol{K}_{\boldsymbol{F} \boldsymbol{T}}}(\boldsymbol{E})_{\boldsymbol{p}} \boldsymbol{\infty}=\mathbf{0} \boldsymbol{?}$ & $\boldsymbol{\mu}_{\boldsymbol{p}, \boldsymbol{1}}^{\text {Per }}$ & $\boldsymbol{\mu}_{\boldsymbol{p}, \mathbf{1}}^{\text {naive }}$ \\
\hline 7 & 2 & $1.7^{0}+3.7^{1}+1.7^{2}+O\left(7^{3}\right)$ & Yes & 4 & $\mathbf{5}$ \\
13 & 2 & $7.13^{0}+5.13^{1}+9.13^{2}+O\left(13^{3}\right)$ & Yes & 10 & 10 \\
19 & - & 0 & No & 17 & 17 \\
31 & - & 0 & No & 29 & 29 \\
37 & - & 0 & No & 33 & 33 \\
43 & 7 & $24.43^{0}+20.43^{1}+1.43^{2}+O\left(43^{3}\right)$ & Yes & 41 & 41 \\
61 & 2 & $16.61^{0}+50.61^{1}+46.61^{2}+O\left(61^{3}\right)$ & Yes & 53 & $\mathbf{5 4}$ \\
67 & 17 & $53.67^{0}+21.67^{1}+52.67^{2}+O\left(67^{3}\right)$ & Yes & 61 & 61 \\
73 & 2 & $13.73^{2}+13.73^{4}+20.73^{5}+O\left(73^{6}\right)$ & No & 64 & $\mathbf{6 6}$ \\
79 & 2 & $50.79^{0}+77.79^{1}+55.79^{2}+O\left(79^{3}\right)$ & Yes & 75 & $\mathbf{7 7}$ \\
97 & 2 & $87.97^{2}+88.97^{3}+29.97^{4}+O\left(97^{5}\right)$ & No & 94 & 94 \\
\hline
\end{tabular}

TABLE 1. The elliptic curve $E=27 A(1): y^{2}+y=x^{3}$.

\begin{tabular}{|c|c|c|c|c|c|}
\hline $\boldsymbol{p}$ & $\boldsymbol{\Delta}$ & $\boldsymbol{\mathcal { X }}_{\boldsymbol{E}}\left(\mathbf{R e g}_{\mathbb{Q}\left(\boldsymbol{\mu}_{\boldsymbol{p}}\right) / \mathbb{Q}}\right)$ & $\mathbf{S e l}_{\boldsymbol{K}_{\boldsymbol{F} \boldsymbol{T}}}(\boldsymbol{E})_{\boldsymbol{p}} \boldsymbol{\infty}=\mathbf{0} \boldsymbol{?}$ & $\boldsymbol{\mu}_{\boldsymbol{p}, \boldsymbol{1}}^{\text {Per }}$ & $\boldsymbol{\mu}_{\boldsymbol{p}, \mathbf{1}}^{\text {naive }}$ \\
\hline 5 & - & 0 & No & 3 & 3 \\
13 & - & 0 & No & 11 & 11 \\
17 & - & 0 & No & 14 & 14 \\
29 & - & 0 & No & 27 & 27 \\
37 & - & 0 & No & 34 & 34 \\
41 & - & 0 & No & 38 & 38 \\
53 & - & 0 & No & 51 & 51 \\
61 & - & 0 & No & 56 & $\mathbf{5 8}$ \\
73 & 3 & $35.73^{0}+26.73^{1}+61.73^{2}+O\left(73^{3}\right)$ & Yes & 68 & 68 \\
89 & 3 & $67.89^{0}+13.89^{1}+59.89^{2}+O\left(89^{3}\right)$ & Yes & 82 & $\mathbf{8 4}$ \\
97 & 3 & $9.97^{0}+2.97^{1}+29.97^{2}+O\left(97^{3}\right)$ & Yes & 94 & 94 \\
\hline
\end{tabular}

TABLE 2. The elliptic curve $E=32 A(1): y^{2}=x^{3}+4 x$.

\begin{tabular}{|c|c|c|c|c|c|}
\hline $\boldsymbol{p}$ & $\boldsymbol{\Delta}$ & $\boldsymbol{\mathcal { X }}_{\boldsymbol{E}}\left(\mathbf{R e g}_{\mathbb{Q}\left(\boldsymbol{\mu}_{\boldsymbol{p}}\right) / \mathbb{Q}}\right)$ & $\mathbf{S e l}_{\boldsymbol{K}_{\boldsymbol{F} \boldsymbol{T}}}(\boldsymbol{E})_{\boldsymbol{p}} \boldsymbol{\infty}=\mathbf{0} \boldsymbol{?}$ & $\boldsymbol{\mu}_{\boldsymbol{p}, \mathbf{1}}^{\text {Per }}$ & $\boldsymbol{\mu}_{\boldsymbol{p}, \mathbf{1}}^{\text {naive }}$ \\
\hline 11 & - & 0 & No & 6 & $\mathbf{8}$ \\
23 & - & 0 & No & 20 & 20 \\
29 & 3 & $18.29^{0}+2.29^{1}+10.29^{2}+O\left(29^{3}\right)$ & Yes & 25 & 25 \\
37 & 5 & $32.37^{0}+10.37^{1}+22.37^{3}+O\left(37^{4}\right)$ & Yes & 33 & 33 \\
43 & - & 0 & No & 24 & $\mathbf{2 8}$ \\
53 & - & 0 & No & 44 & $\mathbf{4 6}$ \\
67 & - & 0 & No & 61 & $\mathbf{6 3}$ \\
71 & - & 0 & No & 66 & $\mathbf{6 8}$ \\
79 & - & 0 & No & 76 & 76 \\
107 & - & 0 & No & 104 & 104 \\
109 & 2 & $39.109^{2}+34.109^{3}+O\left(109^{4}\right)$ & No & 105 & 105 \\
\hline
\end{tabular}

TABLE 3. The elliptic curve $E=49 A(1): y^{2}+x y=x^{3}-x^{2}-2 x-1$. 
of the symmetric square for the base-change of $f_{E} / K_{n}^{+}$. Following renormalization, one deduces that

$$
\left\langle\theta\left(\lambda_{\mathcal{P}}\right) \otimes \eta_{\mathcal{P}}^{\prime}, \theta\left(\lambda_{\mathcal{P}}\right) \otimes \eta_{\mathcal{P}}^{\prime}\right\rangle_{N}
$$

is equal to

$$
\left|\operatorname{Disc}\left(K_{n}^{+}\right)\right|_{\infty} \pi^{-\left[K_{n}^{+}: \mathbb{Q}\right]-\{2\}} \times L^{\mathrm{imp}}\left(\operatorname{Sym}^{2} E / K_{n}^{+}, 2\right)
$$

up to a $p$-adic unit.

Fortunately, $K_{n}^{+}$is an abelian extension of $\mathbb{Q}$, and so the $L$-function decomposes as a product over the characters of $G=\operatorname{Gal}\left(K_{n}^{+} / \mathbb{Q}\right)$. As an immediate consequence,

$$
\begin{aligned}
& \left\langle\theta\left(\lambda_{\mathcal{P}}\right) \otimes \eta_{\mathcal{P}}^{\prime}, \theta\left(\lambda_{\mathcal{P}}\right) \otimes \eta_{\mathcal{P}}^{\prime}\right\rangle_{N} \\
& \quad \approx\left|\operatorname{Disc}\left(K_{n}^{+}\right)\right|_{\infty} \times \prod_{\psi: G \rightarrow \mathbb{C}^{\times}} \pi^{-3} L^{\mathrm{imp}}\left(\operatorname{Sym}^{2} E, \psi, 2\right) \\
& \quad \approx\left|\operatorname{Disc}\left(K_{n}^{+}\right)\right|_{\infty} \times \xi\left(E / K_{n}^{+}\right) \times \prod_{\psi: G \rightarrow \mathbb{C}^{\times}} \frac{\left\langle f_{E}, f_{E}\right\rangle_{N_{E}}}{\tau\left(\psi^{-2}\right)}
\end{aligned}
$$

again up to $p$-adic units.

Remark 3.7. The proof will be finished if we can establish the following:

(a) $\prod_{\psi: G \rightarrow \mathbb{C}^{\times}} \tau\left(\psi^{-2}\right)=\left|\operatorname{Disc}\left(\mathbb{Q}\left(\mu_{p^{n}}\right)^{+}\right)\right|_{\infty} ;$

(b) $\left\langle f_{E}, f_{E}\right\rangle_{N_{E}}=(p$-adic unit $) \times\left(\pi^{-2} \Omega_{E}^{+} \Omega_{E}^{-}\right)$.

On observing that both the degree of $\varphi_{E}: X_{0}\left(N_{E}\right) \rightarrow$ $E$ and its Manin constant $\mathfrak{c}_{\text {Man }}$ are integers coprime to $p$, we find that (b) follows from the well-known identity

$$
\begin{aligned}
\frac{8 \pi^{3}}{N_{E}}\left\langle f_{E}, f_{E}\right\rangle_{N_{E}} & =L^{\mathrm{imp}}\left(\operatorname{Sym}^{2} E, 2\right) \\
& =\frac{\operatorname{deg}\left(\varphi_{E}\right)}{N_{E} \cdot \mathfrak{c}_{\text {Man }}^{2}} \times \pi i \int_{E(\mathbb{C})} \omega_{E} \wedge \overline{\omega_{E}} .
\end{aligned}
$$

To establish claim (a), clearly we must have

$$
\prod_{\psi: G \rightarrow \mathbb{C}^{\times}} \tau\left(\psi^{-2}\right)=\left(\prod_{\psi: G^{2} \rightarrow \mathbb{C}^{\times}} \tau(\bar{\psi})\right)^{2} .
$$

If the automorphism $\sigma \in \operatorname{Gal}\left(K_{n} / \mathbb{Q}\left(\mu_{p^{n}}\right)\right)$ sends $\sqrt{-D}$ to $-\sqrt{-D}$, then

$$
\operatorname{Gal}\left(K_{n} / \mathbb{Q}\right) \cong\langle\sigma\rangle \times \mathbb{F}_{p}^{\times} \times C_{p^{n-1}}
$$

whence

$$
G \cong \frac{\langle\sigma\rangle \times \mathbb{F}_{p}^{\times}}{\langle(\sigma,-1)\rangle} \times C_{p^{n-1}}
$$

Because $p$ is odd, the field cut out by $G^{2} \cong\left(\mathbb{F}_{p}^{\times}\right)^{2} \times C_{p^{n-1}}$ has to be $\mathbb{Q}\left(\mu_{p^{n}}\right) \cap \mathbb{R}$. Via standard properties of Gauss sums and the conductor-discriminant formula,

$$
\begin{aligned}
\prod_{\psi: G^{2} \rightarrow \mathbb{C}^{\times}} \tau(\bar{\psi})^{2} & =\prod_{\psi: G\left(\mathbb{Q}\left(\mu_{p}\right)^{+} / \mathbb{Q}\right) \rightarrow \mathbb{C}^{\times}} \psi(c) \cdot \mathfrak{f}_{\psi} \\
& =\left|\operatorname{Disc}\left(\mathbb{Q}\left(\mu_{p^{n}}\right)^{+}\right)\right|_{\infty}
\end{aligned}
$$

and the result follows.

\section{THE CONNECTION WITH $\Lambda$-MODULES}

Since $p \cdot \mathcal{O}_{K}=\mathfrak{p} \cdot \mathfrak{p}^{*}$ splits over $K$, we choose $\iota_{p}$ such that $\Phi_{E / K}\left(\mathfrak{p}^{*}\right)$ becomes the unit. The Tate module $T_{p}(E)$ of the curve breaks up into $\mathbb{T}_{\mathfrak{p}} \oplus \mathbb{T}_{\mathfrak{p}^{*}}$ over the CM field, with summands $\mathbb{T}_{\mathfrak{p}}=\lim _{n} E\left[\mathfrak{p}^{n}\right]$ and $\mathbb{T}_{\mathfrak{p}^{*}}=\lim _{n} E\left[\mathfrak{p}^{* n}\right]$. It is well known that

$$
\operatorname{Gal}\left(K\left(E\left[p^{\infty}\right]\right) / K\right)=\Delta_{(2)} \times \Gamma_{(2)},
$$

where

$$
\# \Delta_{(2)}=(p-1)^{2} \quad \text { and } \quad \Gamma_{(2)} \cong \mathbb{Z}_{p} \times \mathbb{Z}_{p}
$$

One can pick a decomposition $\Gamma_{(2)}=\Gamma_{+} \times \Gamma_{-}$such that the action of complex conjugation on $\Gamma_{+}$is trivial and its action on $\Gamma_{-}$is through inversion instead. The Iwasawa algebra $\mathbb{Z}_{p}\left[\left[\Gamma_{+} \times \Gamma_{-}\right]\right]$is (noncanonically) isomorphic to $\mathbb{Z}_{p} \llbracket S, T \rrbracket$; here one distinguishes the variables $S$ and $T$ by a choice of topological generators $\gamma_{+} \in \Gamma_{+}$ and $\gamma_{-} \in \Gamma_{-}$respectively.

Finally, let us write $\mathcal{M}_{\infty}$ for the maximal abelian pro$p$-extension of $K\left(E\left[p^{\infty}\right]\right)$ unramified outside the places lying over $\mathfrak{p}$, and set $\mathfrak{X}_{\infty}:=\operatorname{Gal}\left(\mathcal{M}_{\infty} / K\left(E\left[p^{\infty}\right]\right)\right)$.

Proposition 4.1. For all integers $n \geq 1$, we have

$$
\begin{aligned}
\mu_{p, n}^{\mathrm{Per}}(E)= & p^{n-1} \times(p n-n-1)+n-\operatorname{ord}_{p}\left(h^{-}\left(K_{n}\right)\right) \\
& -\nabla_{K_{n}^{+}}^{\mathrm{Sym}^{2} E}+\operatorname{ord}_{p}\left(\frac{\# H^{0}\left(\Gamma_{+}^{p^{n-1}}, \mathcal{Z}_{\infty,+}\right)}{\# H^{1}\left(\Gamma_{+}^{p^{n-1}}, \mathcal{Z}_{\infty,+}\right)}\right) \\
& +k(E, p)
\end{aligned}
$$

where $k=k(E, p)$ is a constant independent of $n$, and the compact $\mathbb{Z}_{p}\left[\left[\Gamma_{+}\right]\right]$-module $\mathcal{Z}_{\infty,+}$ is defined as follows:

$$
\mathcal{Z}_{\infty,+}:=\bigoplus_{j=0}^{p-2} H^{1}\left(\Gamma_{-}, \mathfrak{X}_{\infty} \otimes_{\mathbb{Z}_{p}} \mathbb{T}_{\mathfrak{p}}^{\otimes-2}\right)^{\Delta_{(2)}=\omega_{K}^{j}}
$$

The reader will notice the appearance of the $\left(\Gamma_{+}\right)^{p^{n-1}}-$ Euler characteristic for the $\Phi_{E, \mathfrak{p}}^{\otimes-2}$-twisted coinvariants 
$\mathcal{Z}_{\infty,+}$. The long-term growth in this error function is controlled by its $(\mu, \lambda)$-invariants (discussed in Section 5 at some length).

Henceforth, let us use $\epsilon$ to denote the real quadratic character $\left(\left(\frac{-D}{)}\right) \cdot \omega\right)^{\frac{p-1}{2}}$. We now claim that Proposition 4.1 is a direct consequence of the following two results.

Lemma 4.2. Up to an element of $\mathcal{O}_{\mathbb{C}_{p}}^{\times}$, the quantity $\xi\left(E / K_{n}^{+}\right)$equals

$$
\begin{aligned}
p^{k^{\prime}+\nabla_{K_{n}^{+}}^{\mathrm{Sym}^{2} E}} \times \frac{\left|\operatorname{Disc}\left(\mathbb{Q}\left(\mu_{p^{n}}\right)^{+}\right)\right|_{\infty}}{\left|\operatorname{Disc}\left(K_{n}^{+}\right)\right|_{\infty}^{2}} \\
\times \prod_{\substack{\psi: \operatorname{Gal}\left(K_{n}^{+} / \mathbb{Q}\right) \rightarrow \mathbb{C} \times \\
\psi \neq \mathbf{1}, \psi \neq \epsilon}} \frac{\tau(\psi) L^{\operatorname{prim}}\left(\mathrm{Sym}^{2} E \otimes \psi^{-1}, 1\right)}{\pi \times\left\langle f_{E}, f_{E}\right\rangle_{N_{E}}}
\end{aligned}
$$

for some constant $k^{\prime}$ independent of $n$.

Lemma 4.3. There exists another constant $k^{\prime \prime}$, independent of $n$, such that

$$
\begin{aligned}
& \prod_{\psi \neq \mathbf{1}, \epsilon} \frac{\tau(\psi) L^{\operatorname{prim}}\left(\mathrm{Sym}^{2} E \otimes \psi^{-1}, 1\right)}{\pi \times\left\langle f_{E}, f_{E}\right\rangle_{N_{E}}} \\
& \quad \approx \frac{p^{k^{\prime \prime}} \times h^{-}\left(K_{n}\right)}{\# \mathbb{G}_{m}\left(K_{n}\right)_{\text {tors }}} \times \mathfrak{X}_{\Phi_{E}^{-2}}\left(\Gamma_{+}^{p^{n-1}}\right)^{-1}
\end{aligned}
$$

where we write $\mathfrak{X}_{\Phi_{E}^{-2}}\left(\Gamma_{+}^{p^{n-1}}\right)$ for the ratio

$$
\frac{\# H^{0}\left(\Gamma_{+}^{p^{n-1}}, \mathcal{Z}_{\infty,+}\right)}{\# H^{1}\left(\Gamma_{+}^{p^{n-1}}, \mathcal{Z}_{\infty,+}\right)}
$$

To see why these two lemmas imply our principal result, one simply observes that by Proposition 3.3,

$$
\begin{aligned}
p^{\mu_{p, n}^{\mathrm{Per}}(E)}= & (\text { a } p \text {-adic unit }) \times \frac{\left|\operatorname{Disc}\left(\mathbb{Q}\left(\mu_{p^{n}}\right)^{+}\right)\right|_{\infty}}{\left|\operatorname{Disc}\left(K_{n}^{+}\right)\right|_{\infty}} \\
& \times \xi\left(E / K_{n}^{+}\right)^{-1} .
\end{aligned}
$$

Because the field $K_{n}^{+}$has discriminant equal to $p^{p^{n-1}(p n-n-1)} \times \operatorname{Disc}(K)^{\phi\left(p^{n}\right) / 2}$, and further $\# \mathbb{G}_{m}\left(K_{n}\right)_{\text {tors }}=$ unit $\times p^{n}$, we see that Proposition 4.1 follows on taking $k=-\left(k^{\prime}+k^{\prime \prime}\right)$.

of Lemma 4.2. Let us begin with the trivial comment

$$
\begin{aligned}
\xi\left(E / K_{n}^{+}\right)= & \frac{L^{\mathrm{imp}}\left(\mathrm{Sym}^{2} E / K_{n}^{+}, 2\right)}{L \operatorname{prim}\left(\mathrm{Sym}^{2} E / K_{n}^{+}, 2\right)} \\
& \times \prod_{\psi: G\left(K_{n}^{+} / \mathbb{Q}\right) \rightarrow \mathbb{C}^{\times}} \frac{\tau\left(\psi^{-2}\right) L^{\operatorname{prim}}\left(\mathrm{Sym}^{2} E \otimes \psi, 2\right)}{\pi^{3}\left\langle f_{E}, f_{E}\right\rangle_{N_{E}}}
\end{aligned}
$$

and that the first factor $\frac{L^{\mathrm{imp}}(\ldots)}{L^{\mathrm{prim}}(\ldots)}$ has $p$-adic order equal to $\nabla_{K_{n}^{+}}^{\mathrm{Sym}^{2} E}$ by its definition. If we fix a character $\psi$ of $\operatorname{Gal}\left(K_{n}^{+} / \mathbb{Q}\right)$, the functional equation for $\operatorname{Sym}^{2} E \otimes \psi \mathrm{im}-$ plies

$$
\begin{aligned}
& \frac{\tau\left(\psi^{-2}\right) L^{\operatorname{prim}}\left(\mathrm{Sym}^{2} E \otimes \psi, 2\right)}{\pi^{3}\left\langle f_{E}, f_{E}\right\rangle_{N_{E}}} \\
& \quad=\frac{\tau\left(\psi^{-2}\right) \cdot \tau(\psi)^{2}}{\mathfrak{u}^{(\psi)} \times \mathfrak{f}_{\psi}^{3}} \times \frac{\tau(\psi) L^{\mathrm{prim}}\left(\mathrm{Sym}^{2} E \otimes \psi^{-1}, 1\right)}{\pi \times\left\langle f_{E}, f_{E}\right\rangle_{N_{E}}},
\end{aligned}
$$

where the algebraic number

$$
\mathfrak{u}^{(\psi)}=\frac{1}{2} \times \bar{\psi}\left(\mathfrak{f}_{\mathrm{Sym}^{2} E}\right) \sqrt{\mathfrak{f}_{\mathrm{Sym}^{2} E}}
$$

is a $p$-adic unit.

A straightforward exercise (cf. the proof of Proposition 3.3) shows that

$$
\prod_{\psi: G\left(K_{n}^{+} / \mathbb{Q}\right) \rightarrow \mathbb{C}^{\times}} \frac{\tau\left(\psi^{-2}\right) \cdot \tau(\psi)^{2}}{\mathfrak{u}_{\psi} \times \mathfrak{f}_{\psi}^{3}} \approx \frac{\left|\operatorname{Disc}\left(\mathbb{Q}\left(\mu_{p^{n}}\right)^{+}\right)\right|_{\infty}}{\left|\operatorname{Disc}\left(K_{n}^{+}\right)\right|_{\infty}^{2}}
$$

so putting

$$
k^{\prime}=\sum_{\psi=\mathbf{1}, \epsilon} \operatorname{ord}_{p}\left(\frac{\tau(\psi) L^{\mathrm{prim}}\left(\mathrm{Sym}^{2} E \otimes \psi^{-1}, 1\right)}{\pi \times\left\langle f_{E}, f_{E}\right\rangle_{N_{E}}}\right),
$$

the result follows.

of Lemma 4.3. The statement of this lemma is much deeper. The key point is that for $\psi \neq \mathbf{1}, \epsilon$ one has

$$
\begin{gathered}
\Phi_{E / K}\left(\mathfrak{p}^{*}\right)^{-2 \mathfrak{f}_{\psi_{p}}} \times \frac{\tau(\psi) L^{\mathrm{prim}}\left(\mathrm{Sym}^{2} E \otimes \psi^{-1}, 1\right)}{\pi \times\left\langle f_{E}, f_{E}\right\rangle_{N_{E}}} \\
=\int_{g \in G\left(K_{\infty}^{+} / \mathbb{Q}\right)} \bar{\psi}(g) \cdot \mathrm{d} \tau_{\mathrm{Sym}^{2} E}(g),
\end{gathered}
$$

where $\tau_{\mathrm{Sym}^{2} E}$ denotes the measure attached to the symmetric square of $E$ at $s=1$. Moreover, the formal identity

$$
\begin{aligned}
& L^{\operatorname{prim}}\left(\operatorname{Sym}^{2} E \otimes \psi^{-1}, s\right) \\
&= L\left(\psi^{-1} \cdot\left(\frac{-D}{-D}\right), s-1\right) \\
& \quad \times L\left(\Phi_{E / K}^{2} \cdot\left(\psi^{-1} \circ N_{K / \mathbb{Q}}\right), s\right)
\end{aligned}
$$

forces the distribution $\tau_{\mathrm{Sym}^{2} E}$ to fracture into the convolution of a 1-dimensional and a 2-dimensional component. 
Remark 4.4. It was shown in [Coates and Schmidt 87, Propositions 5.7 and 5.13] that

$$
\begin{aligned}
& \int_{G\left(K_{\infty}^{+} / \mathbb{Q}\right)} \psi \cdot \mathrm{d} \tau_{\mathrm{Sym}^{2} E} \\
& =I_{\vartheta} \times u_{\vartheta}\left(\operatorname{Res}(\psi)\left(\gamma_{+}\right)-1\right) \times \Omega_{\mathfrak{p}}^{-2} \times L\left(\psi \cdot\left(\frac{-D}{}\right), 0\right) \\
& \quad \times\left.\operatorname{char}_{\mathbb{Z}_{p} \llbracket S \rrbracket}\left(\left(\mathfrak{X}_{\infty, \vartheta} \otimes_{\mathbb{Z}_{p}} \mathbb{T}_{\mathfrak{p}}^{\otimes-2}\right)_{\Gamma_{-}}\right)\right|_{S=\operatorname{Res}(\psi)\left(\gamma_{+}\right)-1},
\end{aligned}
$$

where $\vartheta=\left.\Phi_{E, \mathfrak{p}}^{2} \cdot \operatorname{Res}(\psi)\right|_{\Delta^{(2)}}$, the series $u_{\vartheta}(S)$ belongs to $\mathbb{Z}_{p} \llbracket S \rrbracket^{\times}$, and the algebraic number $I_{\vartheta}$ is equal to

$$
\frac{\left(\pi^{-1} \times \Omega\right)^{2}}{\left\langle f_{E}, f_{E}\right\rangle_{N_{E}}} \times \frac{\sqrt{\operatorname{Disc}(K) \cdot \operatorname{Norm}_{K / \mathbb{Q}}\left(\mathfrak{f}_{\Phi_{E / K}^{2}}\right)}}{24 \times W\left(\bar{\Phi}_{E / K}^{2}\right)} .
$$

In fact, Coates and Schmidt consider the Lie group $\operatorname{Gal}\left(\mathbb{Q}\left(\mu_{p^{\infty}}\right) / \mathbb{Q}\right)$ instead of $\operatorname{Gal}\left(K\left(\mu_{p^{\infty}}\right)^{+} / \mathbb{Q}\right)$, but the details are otherwise identical. Of course, at the time their article was published, the two-variable main conjecture had not yet been proven; however, thanks to the fundamental work of [Rubin 99], this formula is now unconditional.

Without bothering to explain the various terms constituting $I_{\vartheta}$, we point out that it is a unit whenever $p>3$ does not divide the degree of the modular parameterization. By the same token, the $p$-adic period $\Omega_{\mathfrak{p}}$ always belongs to $\mathcal{O}_{\mathbb{C}_{p}}^{\times}$in the $\mathrm{CM}$ scenario.

Consider the module

$\mathfrak{X}_{\infty}^{\prime \prime}:=H^{0}\left(\Delta_{(2)},\left(\mathfrak{X}_{\infty} \oplus\left(\mathfrak{X}_{\infty} \otimes \omega_{K}^{(p-1) / 2}\right)\right) \otimes_{\mathbb{Z}_{p}} \mathbb{T}_{\mathfrak{p}}^{\otimes-2}\right)$.

The proof of Lemma 4.3 reduces to proving the following two statements:

\section{Fact 4.5.}

$$
\begin{aligned}
& \prod_{\psi \neq \mathbf{1}, \epsilon} L\left(\psi \cdot\left(\frac{-D}{)}\right), 0\right) \\
& \quad \approx \frac{h^{-}\left(K_{n}\right)}{\# \mathbb{G}_{m}\left(K_{n}\right)_{\mathrm{tors}}} \times \frac{1}{L\left(\left(\frac{-D}{)}\right), 0\right) \cdot L\left(\epsilon \cdot\left(\frac{-D}{}\right), 0\right)} .
\end{aligned}
$$

\section{Fact 4.6.}

$$
\begin{aligned}
& \mathfrak{X}_{\Phi_{E}^{-2}}\left(\Gamma_{+}^{p^{n-1}}\right) \\
& \quad \times\left.\prod_{\psi \neq \mathbf{1}, \epsilon} \operatorname{char}_{\mathbb{Z}_{p} \llbracket S \rrbracket}\left(\left(\mathfrak{X}_{\infty, \vartheta} \otimes_{\mathbb{Z}_{p}} \mathbb{T}_{\mathfrak{p}}^{\otimes-2}\right)_{\Gamma_{-}}\right)\right|_{S=\psi_{K}\left(\gamma_{+}\right)-1} \\
& \quad \approx \# H^{0}\left(\Gamma_{+},\left(\mathfrak{X}_{\infty}^{\prime \prime}\right)_{\Gamma_{-}}\right) / \# H^{1}\left(\Gamma_{+},\left(\mathfrak{X}_{\infty}^{\prime \prime}\right)_{\Gamma_{-}}\right) .
\end{aligned}
$$

If we can prove these statements, Lemma 4.3 will hold for the constant

$$
\begin{aligned}
k^{\prime \prime}= & \operatorname{ord}_{p}\left(\frac{\# H^{0}\left(\Gamma_{+},\left(\mathfrak{X}_{\infty}^{\prime \prime}\right)_{\Gamma_{-}}\right)}{\# H^{1}\left(\Gamma_{+},\left(\mathfrak{X}_{\infty}^{\prime \prime}\right)_{\Gamma_{-}}\right)}\right) \\
& -\operatorname{ord}_{p}\left(L\left(\left(\frac{-D}{-D}\right), 0\right) \cdot L\left(\epsilon \cdot\left(\frac{-D}{-}\right), 0\right)\right) .
\end{aligned}
$$

To prove Fact 4.5, we use (the odd part of) the analytic class number formula

$$
\begin{aligned}
& \prod_{\psi: G\left(K_{n}^{+} / \mathbb{Q}\right) \rightarrow \mathbb{C}^{\times}} L\left(\psi \cdot\left(\frac{-D}{}\right), 1\right) \\
= & \frac{(2 \pi)^{\left[K_{n}^{+}: \mathbb{Q}\right]} \times h^{-}\left(K_{n}\right) \times \sqrt{\left|\operatorname{Disc}\left(K_{n}^{+}\right)\right|_{\infty}}}{\mathbf{Q}_{K_{n} / K_{n}^{+}} \times \# \mathbb{G}_{m}\left(K_{n}\right)_{\text {tors }} \times \sqrt{\left|\operatorname{Disc}\left(K_{n}\right)\right|_{\infty}}}
\end{aligned}
$$

and then apply the $\psi \cdot\left(\frac{-D}{}\right)$-twisted functional equation (e.g., see [Washington 96, Theorem 4.17]). Note that the index $\mathbf{Q}_{K_{n} / K_{n}^{+}}$is either 1 or 2 ; hence it plays no role in the calculation.

Remark 4.7. To establish that Fact 4.6 is true, let us assume that $\mathcal{W}$ denotes some compact finitely generated $\mathbb{Z}_{p}\left[\left[\Gamma_{+} \times \Delta_{(2)}\right]\right]$-torsion module. We shall write $\widetilde{\omega}$ to denote the mapping

$$
\Delta_{(2)}=\operatorname{Gal}(K(E[p]) / K) \rightarrow \operatorname{Gal}\left(K\left(\mu_{p}\right) / K\right) \stackrel{\sim}{\longrightarrow} \mathbb{F}_{p}^{\times}
$$

induced by the $p$ th cyclotomic character. Then

$$
\begin{aligned}
& \left.\prod_{\substack{\eta=\operatorname{Res}(\psi), \psi: G\left(K_{n}^{+} / \mathbb{Q}\right) \rightarrow \mathbb{C}_{p}^{\times}}} \operatorname{char}_{\mathbb{Z}_{p} \llbracket S \rrbracket}\left(\mathcal{W}_{\vartheta^{\prime}}\right)\right|_{S=\eta\left(\gamma_{+}\right)-1} \\
= & \left.\prod_{j=0}^{p-2} \prod_{\eta^{\prime}: \Gamma_{+} / \Gamma_{+}^{p^{n-1} \rightarrow \mathbb{C}_{p}^{\times}}} \operatorname{char}_{\mathbb{Z}_{p} \llbracket S \rrbracket}\left(\mathcal{W}_{\widetilde{\omega}^{j}}\right)\right|_{S=\eta^{\prime}\left(\gamma_{+}\right)-1} \\
\approx & \prod_{j=0}^{p-2} \frac{\# H^{1}\left(\Gamma_{+}^{p^{n-1}}, \mathcal{W}_{\widetilde{\omega}^{j}}\right)}{\# H^{0}\left(\Gamma_{+}^{p^{n-1}}, \mathcal{W}_{\widetilde{\omega}^{j}}\right)},
\end{aligned}
$$

provided that each characteristic power series does not vanish at the points $\eta\left(\gamma_{+}\right)-1$. In the previous product, the $\eta$ 's are the restrictions to $G_{K}$ of all even characters

$$
\psi: G_{\mathbb{Q}} \rightarrow \operatorname{Gal}\left(K_{n} / \mathbb{Q}\right) \longrightarrow \mathbb{C}_{p}^{\times},
$$

and the finite character $\vartheta^{\prime}=\left.\eta\right|_{\Delta_{(2)}}$ again means the "restriction of $\eta$ " to the torsion subgroup in $\operatorname{Gal}\left(K\left(E\left[p^{\infty}\right]\right) / K\right)$.

If we now choose $\mathcal{W}=\left(\mathfrak{X}_{\infty} \otimes_{\mathbb{Z}_{p}} \mathbb{T}_{\mathfrak{p}}^{\otimes-2}\right)_{\Gamma_{-}}$, then it is well known to the experts that

$$
\operatorname{char}_{\mathbb{Z}_{p} \llbracket S \rrbracket}\left(\mathcal{W}_{\vartheta^{\prime}}\right)\left(\psi_{K}\left(\gamma_{+}\right)-1\right) \neq 0
$$


for every $\psi_{K}$-twist, since all the special values $L\left(\Phi_{E / K}^{2}\right.$. $\psi_{K}^{-1}, 1$ ) are nonvanishing (see [Coates and Schmidt 87, p. 147] for an explanation). Consequently, the (full) product becomes

$$
\begin{gathered}
\left.\prod_{\psi} \operatorname{char}_{\mathbb{Z}_{p} \llbracket S \rrbracket}\left(\left(\mathfrak{X}_{\infty, \vartheta} \otimes_{\mathbb{Z}_{p}} \mathbb{T}_{\mathfrak{p}}^{\otimes-2}\right)_{\Gamma_{-}}\right)\right|_{S=\operatorname{Res}(\psi)\left(\gamma_{+}\right)-1} \\
=\left.\prod_{\eta=\operatorname{Res}(\psi)} \operatorname{char}_{\mathbb{Z}_{p} \llbracket S \rrbracket}\left(\mathcal{W}_{\vartheta^{\prime}}\right)\right|_{S=\eta\left(\gamma_{+}\right)-1} \\
\underset{\text { by Remark } 4.7}{\approx} \prod_{j=0}^{p-2} \frac{\# H^{1}\left(\Gamma_{+}^{p^{n-1}}, \mathcal{W}_{\widetilde{\omega}^{j}}\right)}{\# H^{0}\left(\Gamma_{+}^{p^{n-1}}, \mathcal{W}_{\widetilde{\omega}^{j}}\right)},
\end{gathered}
$$

(where the first product is over all $\left.\psi: G\left(K_{n}^{+} / \mathbb{Q}\right) \rightarrow \mathbb{C}_{p}^{\times}\right)$, which is none other than the inverse of $\mathfrak{X}_{\Phi_{E}^{-2}}\left(\Gamma_{+}^{p^{n-1}}\right)$.

Lastly, it is an easy exercise to verify that when we omit the exceptional characters $\psi=\mathbf{1}$ and $\psi=\epsilon$ from the product above, one must adjust by the same factor as in the statement of Fact 4.6.

\section{ASYMPTOTIC GROWTH IN THE CM PERIODS}

Recall that we are trying to derive a formula for the ratio of $\Omega_{K_{n}^{+}}^{\operatorname{mot}}(E)$ to $\Omega_{K_{n}^{+}}^{\text {aut }}(E)$. Using Proposition 4.1, one knows that its $p$-adic order $\mu_{p, n}^{\mathrm{Per}}(E)$ is equal to

$$
\begin{aligned}
& p^{n-1} \times(p n-n-1)+n-\operatorname{ord}_{p}\left(h^{-}\left(K_{n}\right)\right)-\nabla_{K_{n}^{+}}^{\mathrm{Sym}^{2} E} \\
& \quad+\operatorname{ord}_{p}\left(\mathfrak{X}_{\Phi_{E}^{-2}}\left(\Gamma_{+}^{p^{n-1}}\right)\right)
\end{aligned}
$$

up to some fixed constant $k=k(E, p)$.

Therefore, to complete the proof of Theorem 1.8, we must prove the following result.

Proposition 5.1. If the $\mu_{\omega^{j}}\left(\mathcal{Z}_{\infty,+}\right)$-invariants simultaneously vanish at every $j$, one has the growth estimate

$$
\mathfrak{X}_{\Phi_{E}^{-2}}\left(\Gamma_{+}^{p^{n-1}}\right)=(\text { a constant }) \times \prod_{j=0}^{p-2} p^{-n \times \lambda_{\omega^{j}}\left(\mathcal{Z}_{\infty,+}\right)}
$$

for integers $n \gg 1$.

Proof. Again we set $\mathcal{W}=\left(\mathfrak{X}_{\infty} \otimes_{\mathbb{Z}_{p}} \mathbb{T}_{\mathfrak{p}}^{\otimes-2}\right)_{\Gamma_{-}}$, so from the precise definition of $\mathcal{Z}_{\infty,+}$,

$$
\begin{aligned}
\mathfrak{X}_{\Phi_{E}^{-2}}\left(\Gamma_{+}^{p^{n-1}}\right)= & \frac{\# H^{0}\left(\Gamma_{+}^{p^{n-1}}, \bigoplus_{j=0}^{p-2} \mathcal{W}_{\widetilde{\omega}^{j}}\right)}{\# H^{1}\left(\Gamma_{+}^{p^{n-1}}, \bigoplus_{j=0}^{p-2} \mathcal{W}_{\widetilde{\omega}^{j}}\right)} \\
& =\prod_{j=0}^{p-2} \frac{\# H^{0}\left(\Gamma_{+}^{p^{n-1}}, \mathcal{W}_{\widetilde{\omega}^{j}}\right)}{\# H^{1}\left(\Gamma_{+}^{p^{n-1}}, \mathcal{W}_{\widetilde{\omega}^{j}}\right)}
\end{aligned}
$$

Focusing first on the $H^{1}$-term, for a given value $j \in$ $\{0, \ldots, p-2\}$, we have

$$
\begin{aligned}
\# H^{1}\left(\Gamma_{+}^{p^{n-1}}, \mathcal{W}_{\widetilde{\omega}^{j}}\right) & =\#\left(\mathcal{W}_{\widetilde{\omega}^{j}}\right)_{\Gamma_{+}^{p^{n-1}}} \\
& =p^{\lambda_{\omega j} \times n+p^{n} \times \mu_{\omega}+k_{j}^{\prime \prime \prime}}
\end{aligned}
$$

with $n \gg 1$, where the nonnegative integer $k_{j}^{\prime \prime \prime}$ is independent of $n$.

Remark 5.2. We now exploit two important facts:

(i) The Iwasawa $\mu$-invariants of the $\mathbb{Z}_{p} \llbracket \Gamma_{+} \rrbracket$-modules $\mathcal{W}_{\widetilde{\omega}^{j}}$ are assumed trivial.

(ii) All finite $\mathbb{Z}_{p} \llbracket \Gamma_{+} \rrbracket$-submodules of $\mathcal{W}_{\widetilde{\omega}^{j}}$ have universally bounded size.

We note that the first statement clearly implies the vanishing of the total $\mu_{E}^{\mathrm{cy}}$-invariant of $\mathcal{W}$. To see why (i) implies (ii), there is an exact sequence of finitely generated compact $\mathbb{Z}_{p} \llbracket \Gamma_{+} \rrbracket$-modules

$$
\begin{aligned}
& 0 \longrightarrow \text { finite } \longrightarrow \mathcal{W}_{\widetilde{\omega}^{j}} \stackrel{\mathfrak{h}_{j}}{\longrightarrow} \bigoplus_{m=1}^{t_{j}} \mathbb{Z}_{p} \llbracket \Gamma_{+} \rrbracket /\left(F_{j, m}\right)^{e_{j, m}} \\
& \longrightarrow \text { finite } \longrightarrow 0
\end{aligned}
$$

where the $F_{j, m}$ 's map to irreducible distinguished polynomials via $\mathbb{Z}_{p} \llbracket \Gamma_{+} \rrbracket \stackrel{\sim}{\longrightarrow} \mathbb{Z}_{p} \llbracket S \rrbracket$. As a corollary, the size of any pseudonull $\mathbb{Z}_{p} \llbracket \Gamma_{+} \rrbracket$-submodule inside $\mathcal{W}_{\widetilde{\omega}^{j}}$ must be bounded above independently by $\# \operatorname{Ker}\left(\mathfrak{h}_{j}\right)$, since the summands $\mathbb{Z}_{p} \llbracket \Gamma_{+} \rrbracket /\left(F_{j, m}\right)^{e_{j, m}}$ are easily seen to be free of any $p^{\infty}$-torsion (which establishes the second fact).

A nice consequence of (ii) is the boundedness of $H^{0}\left(\Gamma_{+}^{p^{n-1}}, \mathcal{W}_{\widetilde{\omega}^{j}}\right)$ for $n \geq 1$, since this module is contained within its $\operatorname{Gal}\left(K_{n}^{+} / K_{1}^{+}\right)$-orbit (which is then a finite $\mathbb{Z}_{p} \llbracket \Gamma_{+} \rrbracket$-submodule of $\left.\mathcal{W}_{\widetilde{\omega}^{j}}\right)$. It follows that $\# H^{0}\left(\Gamma_{+}^{p^{n-1}}, \mathcal{W}_{\widetilde{\omega}^{j}}\right)=p^{k_{j}^{\prime \prime \prime \prime}}$ (say) for $n$ sufficiently large, in which case

$$
\mathfrak{X}_{\Phi_{E}^{-2}}\left(\Gamma_{+}^{p^{n-1}}\right)=\left(p^{\sum_{j} k_{j}^{\prime \prime \prime \prime}-k_{j}^{\prime \prime \prime}}\right) \times \prod_{j=0}^{p-2} p^{-n \times \lambda_{\omega j}\left(\mathcal{Z}_{\infty,+}\right)},
$$

as predicted by Proposition 5.1. The proof is complete.

Question 5.3. Does the vanishing of the total $\mu_{E}^{\text {cy }}$ invariant occur very frequently?

As Ralph Greenberg pointed out to one of us, if we take a two-variable power series (with trivial $\mu$-invariant) 
and consider all of its one-variable specializations, then the subset of specializations with positive $\mu$-invariant should have density zero. Therefore, intuitively we would expect the answer to be yes.

The vanishing of the cyclotomic $\mu$-invariant inside a two-variable deformation, but without the extra twisting by the Grössencharakter $\Phi_{E}^{-2}$, was shown in [Gillard 85, Theorem 3.4]. Similarly, some recent work of Hida confirms the vanishing of the $\mu$-invariant for many anticyclotomic branches of the Katz $p$-adic $L$-function (which, unfortunately, is of no use here).

Table 1 presents a few numerical calculations of the $\mu_{E}^{\text {cy }}$-invariant for our three specimen elliptic curves: $27 A(1), 32 A(1)$, and $49 A(1)$. Briefly, an upper bound on $\mu_{\omega^{j}}\left(\mathcal{Z}_{\infty,+}\right)$ is given by the $p$-adic order at any special value of the $\widetilde{\omega}^{j}$-branch for the $\Phi_{E, \mathfrak{p}^{-}}^{2}$-twisted Katz-Yager $L$-function (which, one may hope, might often be zero).

\subsection{Computational Difficulties at the Second Layer}

Let us now outline several problems arising in the search for congruences at the higher layer $n=2$. Shifting notation slightly, let $F_{n}$ denote the extension $\mathbb{Q}\left(\mu_{p^{n}}\right)$. Kato's predicted congruence [Kato 05, Section 3.10] over the field $\mathbb{Q}\left(\mu_{p^{2}}, \sqrt[p^{2}]{\Delta}\right)$ is precisely

$$
\begin{aligned}
& \operatorname{Norm}_{1,2}\left(\frac{a_{1}}{\operatorname{Norm}_{0,1}\left(a_{0}\right)}\right)^{p} \times\left(\frac{a_{2} \times \varphi \circ \operatorname{Norm}_{0,1}\left(a_{0}\right)}{\operatorname{Norm}_{0,2}\left(a_{0}\right) \times \varphi\left(a_{1}\right)}\right)^{p^{2}} \\
& \stackrel{? ?}{=} 1 \bmod p^{4} \cdot \mathbb{Z}_{p}\left[\left[U^{(2)}\right]\right],
\end{aligned}
$$

where the $a_{j}$ 's denote the motivic $p$-adic $L$-functions $\mathcal{L}\left(E, \rho_{j}\right)$ for $j=0,1,2$.

Remark 5.4. Evaluating the above at the trivial character $\psi=\mathbf{1}$, then exploiting the basic identity $\mathbf{1} \circ \varphi=\mathbf{1}^{p}=\mathbf{1}$, Kato's prediction simplifies to become

$$
\begin{aligned}
& \left(\frac{\mathcal{X}_{E}\left(\operatorname{Res}_{F_{2}}\left(\rho_{1}\right)\right)}{\mathcal{X}_{E}\left(\operatorname{Res}_{F_{2}}\left(\rho_{0}\right)\right)^{1+p}} \times\left(\mathcal{X}_{E}\left(\rho_{2}\right) \times \frac{\mathcal{X}_{E}\left(\operatorname{Res}_{F_{1}}\left(\rho_{0}\right)\right)}{\mathcal{X}_{E}\left(\rho_{1}\right)}\right)^{p}\right)^{p} \\
& \quad \stackrel{? ?}{=} 1 \bmod p^{4} .
\end{aligned}
$$

Motivated by numerical work in [Dokchitser and Dokchitser 07], we pick our favorite non-CM elliptic curve

$$
E=X_{0}(11): y^{2}+y=x^{3}-x^{2}-10 x-20,
$$

making a choice of the good ordinary prime $p=3$ and also the auxiliary integer $\Delta=2$. Pushing our computer to its limits, we painstakingly calculated the tableau

$$
\begin{aligned}
\mathcal{X}_{E}\left(\operatorname{Res}_{F_{2}}\left(\rho_{1}\right)\right) & =1.3^{0}+2.3^{1}+2.3^{2}+1.3^{3}+2.3^{4}+O\left(3^{5}\right), \\
\mathcal{X}_{E}\left(\operatorname{Res}_{F_{2}}\left(\rho_{0}\right)\right) & =1.3^{0}+2.3^{1}+1.3^{2}+O\left(3^{5}\right), \\
\mathcal{X}_{E}\left(\operatorname{Res}_{F_{1}}\left(\rho_{0}\right)\right) & =1.3^{0}+2.3^{2}+2.3^{3}+1.3^{4}+O\left(3^{5}\right), \\
\mathcal{X}_{E}\left(\rho_{1}\right) & =1.3^{0}+1.3^{2}+1.3^{3}+1.3^{4}+O\left(3^{5}\right) .
\end{aligned}
$$

However the computer failed to work out $\mathcal{X}_{E}\left(\rho_{2}\right)$ due to lack of available memory. This is surprising, given that $X_{0}(11)$ has least conductor among elliptic curves, and likewise $(p, \Delta)=(3,2)$ is pretty much the smallest choice available to us! Nevertheless, if the $K_{1}$-congruence holds over $\mathbb{Q}\left(\mu_{9}, \sqrt[9]{2}\right)$, then one can make an educated guess at the value of $\mathcal{X}_{E}\left(\rho_{2}\right)^{9}$ modulo 81 from the tabulated $L$-values.

Conjecture 5.5. For the elliptic curve $E=X_{0}(11)$, the prime $p=3$, and $\Delta=2$,

$$
\mathcal{X}_{E}\left(\rho_{2}\right) \stackrel{3^{2}}{\stackrel{? ?}{\equiv} 28} \bmod 3^{4} \text {. }
$$

This is equivalent to Kato's second-layer $\mathrm{K}_{1}$ congruence (at the trivial character), and it is easily confirmed that 28 is a ninth power modulo $3^{4}$, e.g., $4^{9} \equiv 28$ $\bmod 3^{4}$. We leave this difficult computation for those more versed in experimental work.

\section{APPENDIX: EVALUATING THE $\nabla_{K_{n}^{+}}^{\mathrm{Sym}^{2} E}$ FACTORS}

We end by describing an algorithm to compute the error $\nabla_{K_{n}^{+}}^{\mathrm{Sym}^{2} E}$ in the CM case, which by definition is the $p$-adic order of the factor $\prod_{q \mid N_{E}} L_{q}\left(\operatorname{Sym}^{2} E / K_{n}^{+}, q^{-2}\right)$. The method generalizes well to adjoint lifts of other nonspherical representations. However, we care only about the weight $(2, \ldots, 2)$ scenario here.

Proposition 6.1. For each rational prime $q \mid N_{E}$, one has the formula

$$
\begin{aligned}
& L_{q}\left(\operatorname{Sym}^{2} E / K_{n}^{+}, q^{-2}\right) \\
& \quad= \begin{cases}\left(1-\varpi q^{-m}\right)^{\phi\left(p^{n}\right) / m}\left(1-q^{-m}\right)^{\phi\left(p^{n}\right) / 2 m} & m \text { odd }, \\
\left(1-|\varpi| q^{-m}\right)^{\phi\left(p^{n}\right) / m}\left(1+q^{-m / 2}\right)^{\phi\left(p^{n}\right) / m} & m \text { even },\end{cases}
\end{aligned}
$$

where $m$ denotes the multiplicative order of $q \in$ $\left(\mathbb{Z} / p^{n} \mathbb{Z}\right)^{\times}$, and $\varpi \in\{-1,0,1\}$ satisfies $L_{q}\left(\operatorname{Sym}^{2} E, X\right)=$ $(1-\varpi q X)$.

The proof of this result is written up in the doctoral dissertation [Ward 09]. Briefly, one partitions the 


\begin{tabular}{|l|c|ccccccccccc|}
\hline $\boldsymbol{E}=\mathbf{2 7} \boldsymbol{A}(\mathbf{1})$ & $\boldsymbol{p}$ & 7 & 13 & 19 & 31 & 37 & 43 & 61 & 67 & 73 & 79 & 97 \\
& $\boldsymbol{\mu}_{\boldsymbol{E}}^{\text {cy }}$ & $\leq 2$ & 0 & 0 & 0 & 0 & 0 & $\leq 2$ & 0 & $\leq 2$ & $\leq 2$ & 0 \\
\hline $\boldsymbol{E}=\mathbf{3 2 A ( 1 )}$ & $\boldsymbol{p}$ & 5 & 13 & 17 & 29 & 37 & 41 & 53 & 61 & 73 & 89 & 97 \\
& $\boldsymbol{\mu}_{\boldsymbol{E}}^{\text {cy }}$ & 0 & 0 & 0 & 0 & 0 & 0 & 0 & $\leq 2$ & 0 & $\leq 2$ & 0 \\
\hline $\boldsymbol{E}=\mathbf{4 9 A} \boldsymbol{A}(\mathbf{1})$ & $\boldsymbol{p}$ & 11 & 23 & 29 & 37 & 43 & 53 & 67 & 71 & 79 & 107 & 109 \\
& $\boldsymbol{\mu}_{\boldsymbol{E}}^{\text {cy }}$ & $\leq 2$ & 0 & 0 & 0 & $\leq 4$ & $\leq 2$ & $\leq 2$ & $\leq 2$ & 0 & 0 & 0 \\
\hline
\end{tabular}

TABLE 4. Some numerical bounds of $\mu_{E}^{c y}$.

character group of $\mathrm{Gal}\left(K_{n}^{+} / \mathbb{Q}\right)$ into even characters $\psi: \operatorname{Gal}\left(\mathbb{Q}\left(\mu_{p^{n}}\right) / \mathbb{Q}\right) \rightarrow \mathbb{C}^{\times}$, and into the family of twists $\psi \otimes\left(\frac{-D}{}\right)$ with $\psi$ odd. Decomposing the $L$-factor above $q$ into these two distinct pieces, the $\psi$-twists yield

$$
\begin{aligned}
& L_{q}\left(\operatorname{Sym}^{2} E / \mathbb{Q}\left(\mu_{p^{n}}\right)^{+}, X\right) \\
& \quad=L_{q}\left(\operatorname{Sym}^{2} H_{p}^{1}(E) \otimes \operatorname{Reg}_{\mathbb{Q}\left(\mu_{p^{n}}\right)^{+} / \mathbb{Q}}, X\right),
\end{aligned}
$$

and similarly, the $\psi \otimes\left(\frac{-D}{}\right)$-twists contribute

$$
\begin{aligned}
& \frac{L_{q}\left(\operatorname{Sym}^{2} E \otimes\left(\frac{-D}{S^{2}}\right) / \mathbb{Q}\left(\mu_{p^{n}}\right), X\right)}{L_{q}\left(\operatorname{Sym}^{2} E \otimes\left(\frac{-D}{)}\right) / \mathbb{Q}\left(\mu_{p^{n}}\right)^{+}, X\right)} \\
& \quad=\frac{L_{q}\left(\operatorname{Sym}^{2} H_{p}^{1}(E) \otimes\left(\frac{-D}{}\right) \otimes \operatorname{Reg}_{\mathbb{Q}\left(\mu_{p^{n}}\right) / \mathbb{Q}}, X\right)}{L_{q}\left(\operatorname{Sym}^{2} H_{p}^{1}(E) \otimes\left(\frac{-D}{}\right) \otimes \operatorname{Reg}_{\mathbb{Q}\left(\mu_{p^{n}}\right)^{+} / \mathbb{Q}}, X\right)} .
\end{aligned}
$$

The proof of our proposition reduces to the following little bit of linear algebra.

Lemma 6.2. Let $V$ be a p-adic representation, and $M / \mathbb{Q}$ a finite Galois extension. Provided the prime $q \neq p$ is unramified in $M / \mathbb{Q}$, we obtain

$$
L_{q}\left(V \otimes \operatorname{Reg}_{M / \mathbb{Q}}, X\right)=\prod_{i}\left(1-\left(\lambda_{i} X\right)^{\mathfrak{f}}\right)^{\mathfrak{r}},
$$

where $L_{q}(V, X)=\prod_{i}\left(1-\lambda_{i} X\right)$, the quantity $\mathfrak{f}$ is the residue degree of $q$ in $M / \mathbb{Q}$, and $\mathfrak{r}$ denotes the number of primes of $M$ lying over $q$.

Observe that the residue class degree of $q$ in the extension $\mathbb{Q}\left(\mu_{p^{n}}\right) / \mathbb{Q}$ is exactly $m$, while the residue class degree of $q$ inside $\mathbb{Q}\left(\mu_{p^{n}}\right)^{+} / \mathbb{Q}$ is $m$ if $m$ is odd, and $m / 2$ if $m$ is even.

Taking $V=\operatorname{Sym}^{2} H_{p}^{1}(E), \quad M=\mathbb{Q}\left(\mu_{p^{n}}\right)^{+}$, then Lemma 6.2 disposes of the even $\psi$-twists. On the other hand, let us take $V=\operatorname{Sym}^{2} H_{p}^{1}(E) \otimes\left(\frac{-D}{}\right)$ and consider $M$ firstly as the CM field $\mathbb{Q}\left(\mu_{p^{n}}\right)$, and secondly as its subfield $\mathbb{Q}\left(\mu_{p^{n}}\right)^{+}$; the $\psi \otimes\left(\frac{-D}{-}\right)$-twists' contribution can be dealt with (using Lemma 6.2 again), because

$$
L_{q}\left(\operatorname{Sym}^{2} H_{p}^{1}(E) \otimes\left(\frac{-D}{)}\right), X\right)=(1-\varpi q X) \times(1-q X) .
$$

On remarking that $\varpi^{m}=\varpi$ when $m$ is odd and $\varpi^{m}=$ $|\varpi|_{\infty}$ when $m$ is even, the truth of Proposition 6.1 follows after some easy algebraic manipulations.

\section{ACKNOWLEDGMENTS}

The authors thank Thanasis Bouganis for informing them of his approach to the Heisenberg-type congruences and for many other suggestions. They are also extremely grateful to Neil Dummigan for his advice on computing the special values of adjoint $L$-series. The first author was supported by an ARC DP1092496 grant.

\section{REFERENCES}

[Bouganis and Dokchitser 07] A. Bouganis and V. Dokchitser. "Algebraicity of $L$-Values for Elliptic Curves in a False Tate Curve Tower." Math. Proc. Camb. Phil. Soc. 142 (2007), 193-204.

[Coates and Perrin-Riou 89] J. Coates and B. Perrin-Riou. "On $p$-adic $L$-Functions Attached to Motives over $\mathbb{Q}$." Adv. Stud. Pure Math. 17 (1989), 23-54.

[Coates and Schmidt 87] J. Coates and C.-G. Schmidt. "Iwasawa Theory for the Symmetric Square of an Elliptic Curve." J. Reine Angew. Math. 375/376 (1987), 104-156.

[Coates et al. 05] J. Coates, T. Fukaya, K. Kato, R. Sujatha, and O. Venjakob. "The $\mathrm{GL}_{2}$ Main Conjecture for Elliptic Curves without Complex Multiplication." Publ. Math. IHES. 101 (2005), 163-208.

[Coates et al. 10] J. Coates, T. Fukaya, K. Kato, and R. Sujatha. "Root Numbers, Selmer Groups and Non-commutative Iwasawa Theory." In preparation, 2010.

[Dokchitser and Dokchitser 07] T. Dokchitser and V. Dokchitser. "Computations in Non-commutative Iwasawa Theory." Proc. London Math. Soc. (3) 94 (2007), 211-272.

[Delbourgo and Ward 08] D. Delbourgo and T. Ward. "Nonabelian Congruences between $L$-Values of Elliptic Curves." Ann. Inst. Fourier, 58:3 (2008), 1023-1055.

[Gillard 85] R. Gillard. "Fonctions $L$-adiques des Corps Quadratiques Imaginaires et de Leurs Extensions Abéliennes." Journal Reine Angew. Math. 358 (1985), 76-91. 
[Hachimori and Venjakob 03] Y. Hachimori and O. Venjakob. "Completely Faithful Selmer Groups over Kummer Extensions." Documenta Mathematica, extra volume in honour of Kazuya Kato's fiftieth birthday (2003), 443478.

[Hida 91] H. Hida. "On $p$-adic $L$-Functions of GL $(2) \times \mathrm{GL}(2)$ over Totally Real Fields." Ann. Inst. Fourier, 41 (1991), 311-391.

[Hida and Tilouine 93] H. Hida and J. Tilouine. "Anticyclotomic Katz $p$-adic $L$-Functions and Congruence Modules." Ann. Sci. École Norm. Sup. 26:4 (1993), 189-259.

[Kato 05] K. Kato. " $K_{1}$ of Some Non-commutative Completed Group Rings." K-Theory 34:2 (2005), 99-140.

[Katz 78] N. M. Katz. "p-adic $L$-Functions for CM Fields." Invent. Math. 49 (1978), 199-297.

[Mazur and Tilouine 90] B. Mazur and J. Tilouine. "Représentations Galoisiennes, Différentielles de Kähler et Conjectures Principales." Publ. Math. IHES. 71 (1990), 65-100.
[Rubin 99] K. Rubin. "Elliptic Curves with Complex Multiplication and the Conjecture of Birch and SwinnertonDyer." In Arithmetic Theory of Elliptic Curves, Lecture Notes in Mathematics 1716, editd by C. Viola, pp. 167234. Berlin: Springer, 1999.

[Stevens 89] G. Stevens. "Stickelberger Elements and the Modular Parametrizations of Elliptic Curves." Inventiones Math. 98:1 (1989), 75-106.

[Sturm 80] J. Sturm. "Special Values of Zeta Functions and Eisenstein Series of Half-Integral Weight." American J. Math. 102 (1980), 219-240.

[Sturm 89] J. Sturm. "Evaluation of the Symmetric Square at the Near Center Point." American J. Math. 111 (1989), $585-598$.

[Tilouine 89] J. Tilouine. "Sur la Conjecture Principale Anticyclotomique." Duke Mathematical Journal 59 (1989), 629673.

[Ward 09] T. Ward. " $K_{1}$-congruences between $L$-values of Elliptic Curves." PhD thesis, University of Nottingham, 2009.

[Washington 96] L. Washington. Introduction to Cyclotomic Fields. New York: Springer, 1996.

Daniel Delbourgo, School of Mathematical Sciences, Monash University, Melbourne, Victoria 3800, Australia (daniel.delbourgo@sci.monash.edu)

Thomas Ward, Department of Mathematics, University of Walk, Bristol BS8 1TW, United Kingdom (maxtw@bristol.ac.uk)

Received December 20, 2008; accepted July 18, 2009. 